
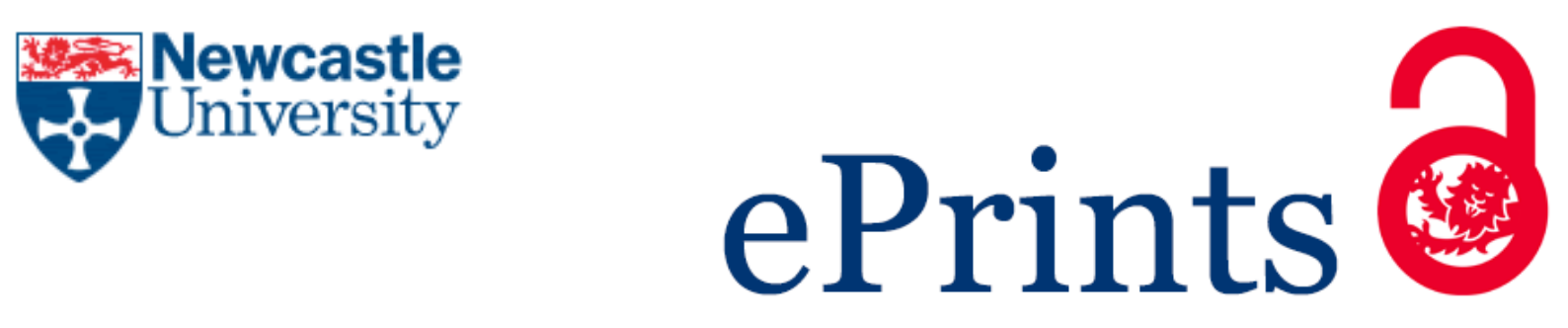

\author{
Chakraborty N, Wang L, Konstantinou I, Klein M. \\ Vorticity statistics based on velocity and density-weighted velocity in \\ premixed reactive turbulence. \\ Journal of Turbulence (2017) \\ DOI: $\underline{\text { https://doi.org/10.1080/14685248.2017.1334905 }}$
}

\title{
Copyright:
}

This is an Accepted Manuscript of an article published by Taylor \& Francis in Journal of Turbulence on 16/06/2017, available online: https://doi.org/10.1080/14685248.2017.1334905

Date deposited:

03/07/2017

Embargo release date:

16 June 2018

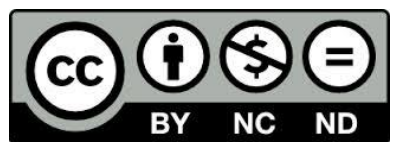

This work is licensed under a

Creative Commons Attribution-NonCommercial-NoDerivatives 4.0 International licence 
Vorticity statistics based on velocity and density-weighted velocity in premixed reactive turbulence

\author{
Nilanjan Chakraborty ${ }^{1}$, Lipo Wang ${ }^{2}$, Ilias Konstantinou ${ }^{1}$, Markus Klein ${ }^{3}$ \\ 1) School of Mechanical and Systems Engineering, Newcastle University, UK \\ ${ }^{2)}$ UM-SJTU Joint Institute, Shanghai JiaoTong University, Shanghai, China \\ ${ }^{3)}$ Universität der Bundeswehr München, Neubiberg, Germany
}

Correspondence details

Email: Lipo.Wang@sjtu.edu.cn

UM-SJTU Joint Institute, Shanghai JiaoTong Univ., 800 DongChuan Rd., 200240, Shanghai, China 


\section{Vorticity statistics based on velocity and density-weighted velocity in premixed reactive turbulence}

Three-dimensional vortex stretching is an inherent feature of turbulent flows. Fundamental understanding of vorticity (i.e. curl of velocity vector $\vec{u}$ ) statistics is therefore of pivotal importance. In the context of combustion, vorticity transport is affected by dilatation rate due to heat release and baroclinic torque. In order to account for variable density in reacting flows the concept of pseudo-vorticity $\vec{\omega}^{*}$ (i.e. curl of density-weighted velocity vector $\rho \vec{u} / \rho_{0}$ with $\rho_{0}$ and $\rho$ being the unburned gas density and local density respectively) has been introduced, and a transport equation for pseudovorticity has been derived in this work. The local and non-local statistics of vorticity $\vec{\omega}$ and pseudo-vorticity $\vec{\omega}^{*}$ have been investigated in detail based on a direct numerical simulation (DNS) database of freely propagating statistically planar flames in different regimes of premixed turbulent combustion. It has been found that the relative orientation of $\vec{\omega}^{*}$ can be significantly different to that of $\vec{\omega}$ for small values of the Karlovitz number and large values of the Reynolds number. The vorticity $\vec{\omega}$ shows, depending on the case, considerable alignment with either the most extensive or compressive principal strain rate. In contrast $\vec{\omega}^{*}$ has been found to align with the intermediate principal strain rate for all cases. The transport statistics of Reynolds averaged enstrophy (i.e. $\bar{\Omega}=\overline{\vec{\omega} \cdot \vec{\omega}} / 2$ ) and pseudo-enstrophy (i.e. $\bar{\Omega}^{*}=\overline{\vec{\omega}^{*} \cdot \vec{\omega}^{*}} / 2$ ) have been investigated in detail. As one moves along the vorticity line passing through a given spatial point there exists a local maximum and a local minimum of (pseudo) vorticity magnitude. These two adjacent extremal points define a vorticity line segment (VLS) structure, which has been used to investigate nonlocal vorticity statistics in turbulent premixed combustion. VLS can be characterized by the segment length and the (pseudo) vorticity magnitude difference across the segment, i.e. $\left(\Delta \omega^{*}\right) \Delta \omega$. Strong dilatation effects tend to increase VLS length, whereas turbulent convection disturbs the flow field resulting in shorter VLS. The joint PDFs between the segment length and $(\Delta \omega) \Delta \omega^{*}$ are found to be symmetric, whereas the joint PDFs between the segment length and the pseudo-vorticity magnitude difference across the VLS in the flame normal direction show significant asymmetry, which is primarily determined by density variation across the flame front and turbulence intensity.

Keywords: Vorticity, enstrophy, vorticity line segment, Direct Numerical Simulations (DNS) 


\section{1: Introduction}

Vorticity, $\vec{\omega}$ defined as curl of velocity vector (i.e. $\vec{\omega}=\nabla \times \vec{u}$ ), is a quantity of fundamental importance in turbulent flows. Its mathematical and topological features have been analysed extensively [1]. In complex turbulent flows, the regions of compression and stretching interact with each other, which leads to lumped distributions of vorticity $\vec{\omega}$. The distribution of vorticity is closely related to intermittency in turbulent flows [2]. Vorticity on average is more likely to be stretched than compressed $[3,4]$. Vortex stretching is responsible for spreading turbulent velocity fluctuations over different length scales [5,6]. This stretching mechanism is important for the understanding of the enstrophy (i.e. $\Omega=\vec{\omega} \cdot \vec{\omega} / 2$ ) transport and energy cascade in turbulent flows. Due to stability reasons strong vorticity regions are organised in the form of tubes. Ruetsch and Maxey [7] demonstrated that the dissipation of energy is correlated with vorticity tubes. The regions of moderate dissipation surround the vorticity tubes and regions of intense dissipation exist between two or more neighbouring tubes. Generally large dissipation regions do not coincide with high vorticity regions [2,8]. Numerical data shows various evolution modes from the interaction of spiral sheets and vortex tubes [9]. In describing the vortex tube geometry, general estimation suggests that the length and radius of the vortex tubes are of the order of the Taylor and dissipative scales, respectively [10]. More detailed analysis suggests that the radius of curvature of the vortex filaments remains of the order of the Taylor micro-scale [11]. The correlation length of different properties along the filament is scaled differently with ambiguity [12]. There is still no consensus on the methodology of identification of the vortical structure $[13,14]$. One needs a predetermined threshold value of the second invariant of the velocity gradient tensor to quantify the vortex tubes, which occupy volumetrically a small (i.e. typically less than 
$10 \%)$ portion of the entire turbulent flow field. Haller [12] suggested that the vortex definition should be invariant under a general coordinate transformation, based on which the defined vortical regions under different frames show good agreement. Recently an interesting idea of defining the vortex tube segment [14] has been introduced, which is helpful in quantifying the global statistics of the vorticity field.

In comparison to the analysis of the vorticity field in non-reacting flows, relatively limited focus has been given to turbulent reacting flows, where the underlying flow structure is significantly affected by heat release, density variation and flame normal acceleration. Nomura \& Elgobashi [15], Boratov et al. [16] and Jaberi et al. [17] analysed the alignment of the vorticity vector with local principal strain rates in nonpremixed flames and demonstrated that the vorticity vector $\vec{\omega}$ aligns with the intermediate principal strain rate in non-premixed flames similar to non-reacting turbulent flows. However, the vorticity vector in non-premixed flames shows appreciable probabilities of local alignment with the most extensive principal strain rate [15-17]. Boratov et al. [16] demonstrated that the extent of vorticity alignment with the most extensive principal strain rate increases in the regions where the magnitude of strain rate dominates over the vorticity magnitude. Jaberi et al. [17] indicated that the alignment of vorticity with the most extensive principal strain rate increases due to chemical heat release in non-premixed flames, whereas $\vec{\omega}$ remains mostly perpendicular to the most compressive principal strain rate in both reactive and nonreactive regions of non-premixed turbulent combustion. Hamlington et al. [18] analysed the alignment of vorticity with local principal strain rates in premixed flames in the thin reaction zones regime combustion, and reported that the alignment of $\vec{\omega}$ with local principal strain rates in the thin reaction zones flames is qualitatively similar to the previous findings in the context of non-premixed combustion. Interested readers are 
referred to Chomiak \& Nisbet [19] for the effects of compressibility in turbulent premixed combustion. Recently, Chakraborty [20] revealed that the global Lewis number $L e$ (i.e. ratio of thermal diffusivity to mass diffusivity) can significantly affect the statistical behaviour of vorticity alignment with local principal strain rates. Furthermore, this alignment can be different for the corrugated flamelets and thin reaction zones regimes of combustion. Chakraborty [20] demonstrated that $\vec{\omega}$ does not align with the most extensive principal strain rate and shows predominant alignment with the intermediate and the most compressive principal strain rates for high (low) values of Damköhler number (global Lewis number) where the most extensive principal strain rate remains approximately equal to the local dilatation rate within flamelets. This behaviour is different from that observed earlier in premixed [18] and non-premixed [15-17] flames characterized by unity Lewis number or in non-reacting flows.

Considering the vorticity energy, Hamlington et al. [18] showed that enstrophy decays significantly in the burned gas across the flame brush, whereas Treurniet et al. [21] demonstrated an opposite trend for the flames with high density ratio (or heat release parameter). This behaviour has been explained by Lipatnikov et al. [22] by analysing the terms of enstrophy and vorticity transport equation for weakly turbulent premixed flames representing the corrugated flamelets regime. The enstrophy field in turbulent premixed flames using cinema-stereoscopic Particle Image Velocimetry (PIV) measurements of rim-stabilised turbulent premixed flames has been investigated [23-26] and confirmed some of the observations based on Direct Numerical Simulations (DNS).

In addition to the aforementioned local vorticity statistics (e.g. alignment of vorticity with local principal strain rates) of turbulent premixed flames, the interaction between the flame surface and the turbulent fluid motion is fundamentally non-local in nature. Therefore, it is useful to analyse the turbulent reacting flow physics beyond the 
pointwise local statistics. However, the analysis related to non-local geometrical features is relatively scarce. In order to address this gap, the concept of the dissipation element structure was introduced by Wang and Peters [27] in the context of nonreacting passive scalar turbulence. According to dissipation element analysis, each spatial point is associated with a trajectory along which the scalar value changes monotonically along the local scalar gradient. All the spatial points, whose trajectories share the common local extremal points, are collectively referred to as a dissipation element. Such a structure is space-filling and the statistics of the entire turbulent field in principle can be understood in terms of the fine-structure statistics of the dissipation elements. Wang [14,28] extended this idea to the velocity and vorticity vectors. Consequently Wang et al. [29] and Chakraborty et al. [30] applied the streamline segment concept to study the non-local velocity statistics around the premixed flame surface. The advantage of such an analysis is to address the non-local features of turbulent reacting flow fields in a quantitative manner in terms of precisely defined geometrical structures.

In the present work, an alternative vorticity based on the curl of densityweighted velocity (i.e. pseudo-vorticity) is defined to account for density change due to heat release in an explicit manner. The comparison of this new quantity and vorticity is of fundamental interest to understand the flow structure and the interaction between flame and turbulent fluid motion. The analyses is based on three-dimensional Direct Numerical Simulations (DNS) data of freely propagating turbulent premixed flames with a range of different turbulence intensities and flame features. The main objectives of the present analysis are:

1. To derive a transport equation for the pseudo-vorticity and the associated enstrophy (henceforth referred to as pseudo-enstrophy) 


\section{To analyse the statistical nature of enstrophy $\Omega$ and pseudo-enstrophy $\Omega^{*}$ transport}

3. To indicate the effects of density change on the non-local vorticity segment statistics

4. To provide physical explanations for the aforementioned observations.

\section{2: Mathematical background}

The momentum conservation equation for the $i^{\text {th }}$ direction is given by:

$\frac{\partial u_{i}}{\partial t}+u_{j} \frac{\partial u_{i}}{\partial x_{j}}=-\frac{1}{\rho} \frac{\partial p}{\partial x_{i}}+\frac{1}{\rho} \frac{\partial \tau_{i k}}{\partial x_{k}}$

where $u_{i}$ is the $i^{\text {th }}$ component of velocity, $\rho$ is the gas density and $\tau_{i k}=\mu\left(\partial u_{i} / \partial x_{k}+\right.$ $\left.\partial u_{k} / \partial x_{i}\right)-(2 \mu / 3) \delta_{i k}\left(\partial u_{j} / \partial x_{j}\right)$ are the components of the stress tensor. Taking curl of eq. 1 yields the transport equation of the $i^{t h}$ component of vorticity $\omega_{i}=\varepsilon_{i j k} \partial u_{k} /$ $\partial x_{j}:$

$$
\frac{\partial \omega_{i}}{\partial t}+u_{k} \frac{\partial \omega_{i}}{\partial x_{k}}=\underbrace{\omega_{k} \frac{\partial u_{i}}{\partial x_{k}}}_{\left(t_{1}\right)_{i}}-\underbrace{\epsilon_{i j k} \frac{1}{\rho^{2}} \frac{\partial \rho}{\partial x_{j}} \frac{\partial \tau_{k l}}{\partial x_{l}}}_{\left(t_{21}\right)_{i}}+\underbrace{\frac{\epsilon_{i j k}}{\rho} \frac{\partial^{2} \tau_{k l}}{\partial x_{j} \partial x_{l}}}_{\left(t_{22}\right)_{i}} \underbrace{-\omega_{i} \frac{\partial u_{k}}{\partial x_{k}}}_{\left(t_{3}\right)_{i}}+\underbrace{\frac{\epsilon_{i j k}}{\rho^{2} \frac{\partial \rho}{\partial x_{j}} \frac{\partial p}{\partial x_{k}}}}_{\left(t_{4}\right)_{i}}
$$

The term $\vec{t}_{1}$ on the right hand side of eq. 2 is the vortex-stretching term, whereas the terms $\vec{t}_{21}$ and $\vec{t}_{22}$ arise from the misalignment between the gradients of viscous stress and density and the diffusion of vorticity. The third term on the right hand side of eq. 2 (i.e. term $\vec{t}_{3}$ ) is responsible for vorticity destruction by dilatation rate, whereas the last term on right hand side of eq. 2 (i.e. term $\vec{t}_{4}$ ) is responsible for baroclinic effects arising from the misalignment of the density and pressure gradients.

Multiplying $\omega_{i}$ with both sides of eq. 2 yields the transport equation of enstrophy $\Omega=\omega^{2} / 2=\omega_{i} \omega_{i} / 2$ :

$$
\frac{\partial \Omega}{\partial t}+u_{k} \frac{\partial \Omega}{\partial x_{k}}=\underbrace{\omega_{i} \omega_{k} \frac{\partial u_{i}}{\partial x_{k}}}_{T_{1}} \underbrace{\epsilon_{i j k} \omega_{i} \frac{1}{\rho^{2}} \frac{\partial \rho}{\partial x_{j}} \frac{\partial \tau_{k l}}{\partial x_{l}}}_{T_{21}}+\underbrace{\frac{\epsilon_{i j k} \omega_{i}}{\rho} \frac{\partial^{2} \tau_{k l}}{\partial x_{j} \partial x_{l}}}_{T_{22}} \underbrace{-2 \frac{\partial u_{k}}{\partial x_{k}} \Omega}_{T_{3}}+\underbrace{\epsilon_{i j k} \frac{\omega_{i}}{\rho^{2}} \frac{\partial \rho}{\partial x_{j}} \frac{\partial p}{\partial x_{k}}}_{T_{4}}
$$

It is possible to define a pseudo-vorticity in the following manner

$$
\vec{\omega}^{*}=\nabla \times\left(\rho \vec{u} / \rho_{0}\right)=\vec{\omega}_{\|}^{*}+\vec{\omega}_{\times}^{*} \text { where } \vec{\omega}_{\|}^{*}=\rho \vec{\omega} / \rho_{0} \text { and } \vec{\omega}_{\times}^{*}=(\nabla \rho \times \vec{u}) / \rho_{0}
$$


and to decompose $\vec{\omega}^{*}$ in a component $\vec{\omega}_{\|}{ }^{*}$ which is parallel to $\vec{\omega}$ and a component $\vec{\omega}_{\times}^{*}$ which is in general not aligned with $\vec{\omega}_{\|}^{*}$ and $\vec{\omega}$.

The transport equation of $\omega_{i}^{*}$ can be obtained using eq. 2 and the mass conservation equation (i.e. $D \rho / D t+\rho \nabla \cdot \vec{u}=0)$ :

$$
\begin{gathered}
\frac{\partial \omega_{i}^{*}}{\partial t}+u_{k} \frac{\partial \omega_{i}^{*}}{\partial x_{k}}=\frac{\rho}{\rho_{0}} \omega_{k} \frac{\partial u_{i}}{\partial x_{k}}-\omega_{i}^{*} \frac{\partial u_{k}}{\partial x_{k}}-\frac{\rho}{\rho_{0}} \omega_{i} \frac{\partial u_{k}}{\partial x_{k}}+\frac{\epsilon_{i j k}}{\rho_{0}} \frac{\partial^{2} \tau_{k l}}{\partial x_{j} \partial x_{l}} \\
-\frac{\epsilon_{i j k}}{\rho_{0}} u_{k} \frac{\partial u_{l}}{\partial x_{j}} \frac{\partial \rho}{\partial x_{l}}-\epsilon_{i j k} \frac{\rho}{\rho_{0}} u_{k} \frac{\partial^{2} u_{l}}{\partial x_{j} \partial x_{l}}
\end{gathered}
$$

For a detailed derivation see Appendix A. A comparison between eq. 5 and eq. 2 reveals that the transport equation of pseudo-vorticity $\vec{\omega}^{*}$ does not have the torque contributions due to pressure and viscous stress (i.e. the equivalents of $\vec{t}_{21}$ and $\vec{t}_{4}$ are missing in eq. 5). It is possible to derive a transport equation for pseudo enstrophy $\Omega^{*}=$ $\omega^{* 2} / 2=\omega_{i}^{*} \omega_{i}^{*} / 2$ by multiplying both sides of eq. 5 by $\omega_{i}^{*}$ :

$$
\begin{aligned}
\frac{\partial \Omega^{*}}{\partial t}+u_{k} \frac{\partial \Omega^{*}}{\partial x_{k}} & =\frac{\rho}{\rho_{0}} \omega_{i}^{*} \omega_{k} \frac{\partial u_{i}}{\partial x_{k}}-\omega_{i}^{*} \omega_{i}^{*} \frac{\partial u_{k}}{\partial x_{k}}-\frac{\rho}{\rho_{0}} \omega_{i} \omega_{i}^{*} \frac{\partial u_{k}}{\partial x_{k}} \\
& +\frac{\epsilon_{i j k}}{\rho_{0}} \omega_{i}^{*} \frac{\partial^{2} \tau_{k l}}{\partial x_{j} \partial x_{l}}-\frac{\epsilon_{i j k}}{\rho_{0}} \omega_{i}^{*} u_{k} \frac{\partial u_{l}}{\partial x_{j}} \frac{\partial \rho}{\partial x_{l}}-\epsilon_{i j k} \frac{\rho}{\rho_{0}} \omega_{i}^{*} u_{k} \frac{\partial^{2} u_{l}}{\partial x_{j} \partial x_{l}}
\end{aligned}
$$

On Reynolds averaging eq. 3 one gets:

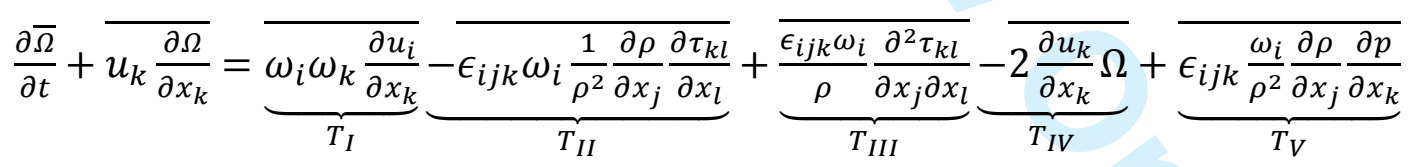

where $\bar{q}(\tilde{q}=\overline{\rho q} / \bar{\rho})$ and $q^{\prime}=q-\bar{q}\left(q^{\prime \prime}=q-\tilde{q}\right)$ are the Reynolds-average (Favreaverage) and Reynolds fluctuation (Favre fluctuation) of a general quantity $q$ respectively. The term $T_{I}$ is the vortex stretching contribution to the mean enstrophy $\bar{\Omega}$ transport, whereas term $T_{I I}$ arises due to misalignment between gradients of density and viscous stresses. The term $T_{I I I}=\overline{(\mu / \rho) \nabla^{2} \Omega}+\overline{(\mu / 3 \rho) \vec{\omega} \cdot[\nabla \times \nabla(\nabla \cdot \vec{u})]}+f(\mu)-D_{v}$ (where $f(\mu)$ represents the contributions from viscosity gradients and $\overline{(\mu / 3 \rho) \vec{\omega} \cdot[\nabla \times \nabla(\nabla \cdot \vec{u})]}$ is identically zero) is responsible for molecular diffusion (i.e. 
$\left.\overline{(\mu / \rho) \nabla^{2} \Omega}+\overline{(\mu / 3 \rho) \vec{\omega} \cdot[\nabla \times \nabla(\nabla \cdot \vec{u})]} \quad \quad\right) \quad$ and $\quad$ dissipation (i.e. $\left.-D_{v}=-\overline{(\mu / \rho)\left(\partial \omega_{l} / \partial x_{l}\right)\left(\partial \omega_{l} / \partial x_{l}\right)}\right)$ of mean enstrophy $\bar{\Omega}$ due to viscous action, whereas the term $T_{I V}$ signifies the enstrophy dilatation contribution. The term $T_{V}$ is the baroclinic torque term which arises due to misalignment between pressure and density gradients. On Reynolds averaging eq. 6 one obtains:

$$
\begin{aligned}
& \frac{\partial \overline{\Omega^{*}}}{\partial t}+\overline{u_{l} \frac{\partial \Omega^{*}}{\partial x_{l}}}=\underbrace{\overline{\frac{\rho}{\rho_{0}} \omega_{i}^{*} \omega_{l} \frac{\partial u_{i}}{\partial x_{l}}}}_{T_{I}^{*}} \underbrace{-\overline{\omega_{i}^{*} \omega_{i}^{*} \frac{\partial u_{l}}{\partial x_{l}}}}_{T_{I I}^{*}} \underbrace{-\overline{\frac{\rho}{\rho_{0}} \omega_{i} \omega_{i}^{*} \frac{\partial u_{l}}{\partial x_{l}}}}_{T_{I I}^{*}} \\
& +\underbrace{\overline{\omega_{i}^{*} \frac{\epsilon_{i j k}}{\rho_{0}} \frac{\partial^{2} \tau_{k l}}{\partial x_{j} \partial x_{l}}}}_{T_{I V}^{*}}-\underbrace{\frac{\overline{\epsilon_{i j k}} u_{\rho_{0}} \omega_{i}^{*} \frac{\partial u_{l}}{\partial x_{j}} \frac{\partial \rho}{\partial x_{l}}}{-\epsilon_{i j k} \frac{\rho}{\rho_{0}} u_{k} \omega_{i}^{*} \frac{\partial^{2} u_{l}}{\partial x_{j} \partial x_{l}}}}_{T_{V}^{*}} \underbrace{\overline{\epsilon_{I}}}_{T_{V I}^{*}}
\end{aligned}
$$

Similar to eq. $7, T_{I}^{*}$ is the vortex stretching contribution to $\overline{\Omega^{*}}$ transport, whereas the terms $T_{I I}^{*}$ and $T_{I I I}^{*}$ signify the dissipation of $\overline{\Omega^{*}}$ due to dilatation. The term $T_{I V}^{*}$ is responsible for molecular diffusion of $\overline{\Omega^{*}}$ due to viscous action, whereas the terms $T_{V}^{*}$ and $T_{V I}^{*}$ originate due to density and dilatation gradients.

The distributions of $\bar{\Omega}$ and $\overline{\Omega^{*}}$ and the relative alignment between $\vec{\omega}^{*}$ and $\vec{\omega}$ will be discussed in Section 4 along with statistical behaviours of $T_{I}-T_{V}$ and $T_{I}^{*}-T_{V I}^{*}$. The first term on the right hand side of eq. 3 is the enstrophy production term which is often expressed as $2 \Omega \partial \vec{u} / \partial s$, where $s$ is the distance in the vorticity line direction. Thus, the statistical behaviour of vorticity lines is of fundamental importance. For this reason, this analysis will focus on the interaction between the flame surface and vorticity lines. The statistical behaviour of the vortex line segments will also be discussed in detail in Section 5 of this paper.

\section{3: Numerical implementation}

Addressing both the three-dimensionality of turbulence and detailed chemical mechanism involves high computational cost for a detailed parametric analysis [31]. 
Thus, in the present study, a simplified single-step Arrhenius type chemical mechanism is considered following several previous analyses [18, 20-22] as the current analysis focuses on fluid-dynamical aspects rather than on chemical details of the flame. In this context, the species field is represented by a reaction progress variable, which can be defined in terms of a suitable reactant mass fraction $Y_{R}$ as: $c=\left(Y_{R 0}-Y_{R}\right) /\left(Y_{R 0}-Y_{R \infty}\right)$ where the subscripts 0 and $\infty$ represent values in unburned reactants and fully burned products respectively. A single step Arrhenius type reaction is taken to represent the chemical reaction. For the present thermo-chemistry the maximum value of reaction rate is obtained close to $c=0.8$, and thus the $c=0.8$ isosurface is considered as the flame surface, following previous studies [29, 30]. For the present analysis, seven freely propagating statistically planar flames (i.e. cases A-G) have been considered, where case $\mathrm{A}$ is taken from an existing DNS database in the literature [32]. Cases B-G are simulated using a well-proven DNS code SENGA [33]. The initial values of the characteristic parameters including the eoot-mean-square (rms) turbulent velocity fluctuation $u^{\prime}$ normalised by the unstrained laminar flame speed $S_{L}$, heat release parameter $\tau=\left(T_{a d}-T_{0}\right) / T_{0}$, Damköhler number $D a=l S_{L} / u^{\prime} \delta_{t h}$ and Karlovitz number $K a=\left(u^{\prime} / S_{L}\right)^{3 / 2}\left(l / \delta_{t h}\right)^{-1 / 2}$ are listed in Table 1 where $\rho_{0}, T_{0}$ and $\mu_{0}$ are the unburned gas density, temperature and viscosity, respectively, $T_{a d}$ is the adiabatic flame temperature, $l$ is the integral scale and $\delta_{t h}=\left(T_{a d}-T_{0}\right) / \max |\nabla \hat{T}|_{L}$ is the thermal flame thickness with $\widehat{T}$ being the instantaneous dimensional temperature. Standard values of Prandtl number $(P r=0.7)$, Zel'dovich number $\left(\beta_{z}=T_{a c}\left(T_{a d}-T_{0}\right) / T_{a d}^{2}=\right.$ 6.0) and the ratio of specific heats $\left(\gamma_{z}=1.4\right)$ were considered for the present analysis where $T_{a c}$ is the activation temperature.

\begin{tabular}{|l|l|l|c|c|c|}
\hline Case & $u^{\prime} / S_{L}$ & $l / \delta_{t h}$ & $\tau$ & $D a$ & $K a$ \\
\hline A & 1.41 & 9.64 & 2.3 & 6.84 & 0.54 \\
\hline B & 7.50 & 2.45 & 3.0 & 0.33 & 13.12 \\
\hline
\end{tabular}




\begin{tabular}{|l|l|l|l|l|l|}
\hline C & 5.00 & 1.67 & 4.5 & 0.33 & 8.67 \\
\hline D & 6.25 & 1.44 & 4.5 & 0.23 & 13.00 \\
\hline E & 7.50 & 2.50 & 4.5 & 0.33 & 13.00 \\
\hline F & 9.00 & 4.31 & 4.5 & 0.48 & 13.00 \\
\hline G & 11.25 & 3.75 & 4.5 & 0.33 & 19.50 \\
\hline
\end{tabular}

Table 1: Initial values of characteristic simulation parameters of all the DNS cases

It can be seen from Table 1 that case A represents the corrugated flamelets $(\mathrm{CF})$ regime (i.e. $K a<1$ ) combustion, whereas combustion in other cases belongs to the thin reaction zones (TRZ) regime. The domain size is taken to be $35.7 \delta_{t h} \times 44.8 \delta_{t h} \times$ $44.8 \delta_{t h}, 24.1 \delta_{t h} \times 24.1 \delta_{t h} \times 24.1 \delta_{t h}$ and $36.1 \delta_{t h} \times 24.1 \delta_{t h} \times 24.1 \delta_{t h}$ for cases A, B and $\mathrm{C}-\mathrm{G}$ respectively. The computational domain is discretised by a uniform Cartesian mesh of $261 \times 128 \times 128,230 \times 230 \times 230$ and $345 \times 230 \times 230$ cells respectively. The long side of the computational domain (i.e. $x_{1}$-direction) is taken to align with the mean direction of flame propagation. The grid spacing is determined by the resolution of flame thickness and about 10 grid points are kept within $\delta_{t h}$. For case A, the boundaries in the direction of mean flame propagation are taken to be inlet and outlet, whereas these boundaries are taken to be partially non-reflecting for cases B-G. The transverse boundaries are taken to be periodic. All non-periodic boundaries are specified using the Navier Stokes Characteristic Boundary Conditions (NSCBC) technique [34]. The spatial discretisation has been carried out using high order finite difference techniques, whereas the time advancement has been carried out using a $3^{\text {rd }}$ order low storage Runge-Kutta technique. The initial turbulent velocity fluctuations were specified using a standard pseudo-spectral method [35], whereas scalar fields were initialised using a steady unstrained planar laminar premixed flame solution. In all cases flame-turbulence interaction takes place under decaying turbulence, which necessitates a simulation time $t_{\operatorname{sim}} \geq \operatorname{Max}\left(t_{f}, t_{c}\right)$, where $t_{f}=l / u^{\prime}$ is the initial eddy turn over time and $t_{c}=\delta_{t h} / S_{L}$ is the chemical time scale. For case A the statistics were extracted after 
$4 t_{f}$, which corresponds to $27 t_{c}$. In cases B-G turbulence is decaying and statistics were extracted after one chemical time scale $t_{c}$, which corresponds to a time equal to $2.0 t_{f}$ in case F, $3.0 t_{f}$ in cases $\mathrm{B}, \mathrm{D}, \mathrm{E}$ and $\mathrm{G}$, and $4.34 t_{f}$ for case $\mathrm{C}$ respectively. The present simulation time is comparable to several previous DNS studies [36-42]. By the time the statistics were extracted, the global turbulent kinetic energy and its dissipation rate in the unburned gas ahead of the flame had decayed by about 50\%, 50\%, 53\%, 61\%, 45\%, $24 \%$ and $34 \%$ in comparison to the initial values for cases A-G respectively, and were no longer changing rapidly with time. By contrast, the integral length scale increased by the factors ranging from 1.5 to 2.25 for case A-G, ensuring that sufficient numbers of turbulent eddies were retained in each direction to obtain useful statistics. It is worth noting that $K a$ remains smaller than unity and the thermal flame thickness $\delta_{\text {th }}$ is smaller than the Kolmogorov length scale $\eta$ at the time of analysis, suggesting that the combustion takes place in the corrugated flamelets regime for case A. The opposite holds true for cases B-G belonging to the thin reaction zones regime. The Reynolds/Favre averaged quantities are evaluated by ensemble averaging the quantities over the plane normal to the mean direction of flame propagation at a given location. Interested readers are referred to Rutland \& Cant [32]; Chakraborty et al. [43-44] for further numerical details.

(a)

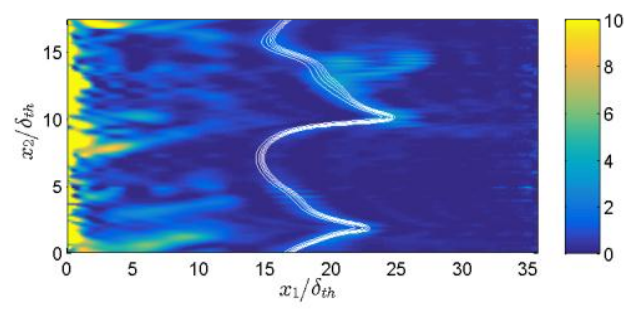

(c) (b)

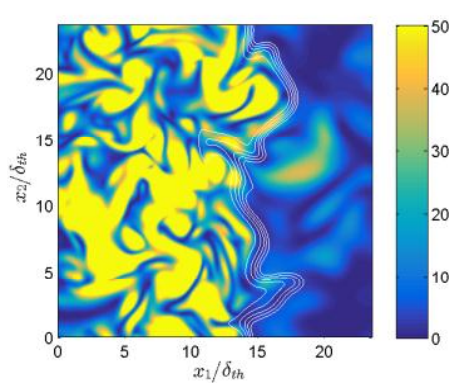

(d) 

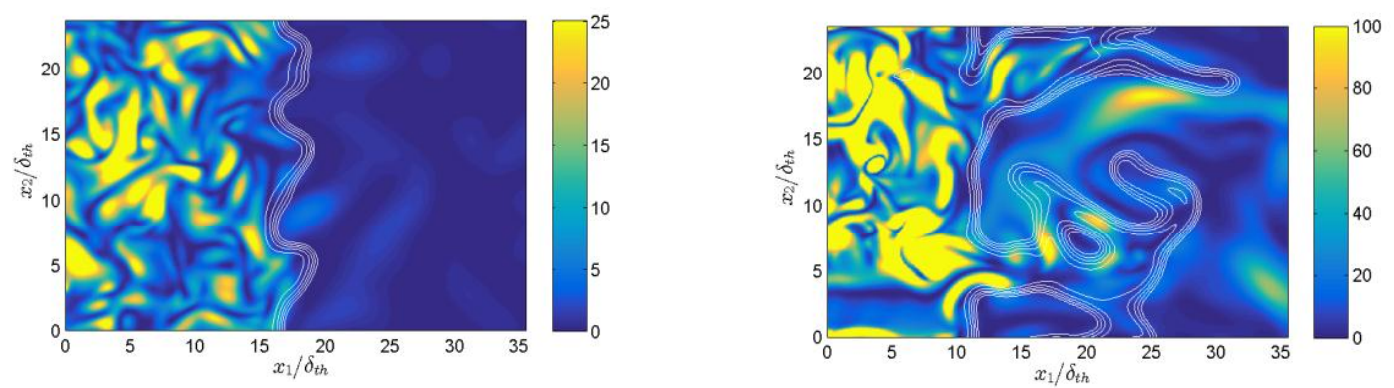

FIGURE 1. Distribution of $\Omega \times \delta_{t h}^{2} / S_{L}^{2}$ in the central $x_{1}-x_{2}$ plane when statistics have been extracted for (a) case A; (b) case B; (c) case C and (d) case G. For the sake of brevity, other case results are not presented. The contours of $c$ from 0.1 to 0.9 in steps of 0.2 (left to right) are shown by white lines.

\section{4: Local statistical properties}

(a)

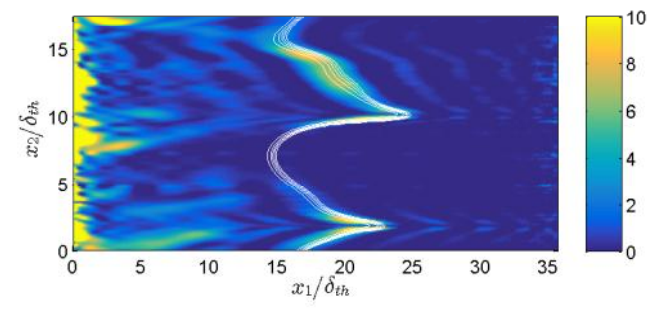

(c)

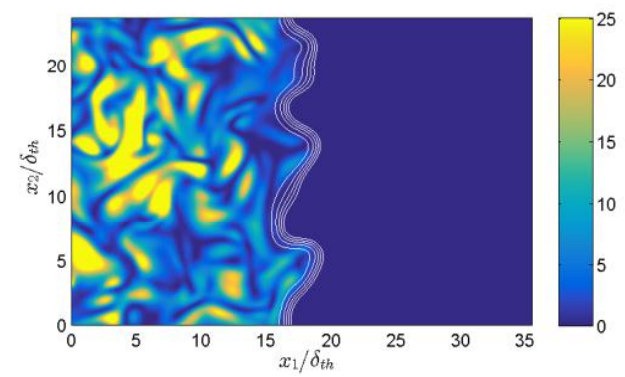

(b)

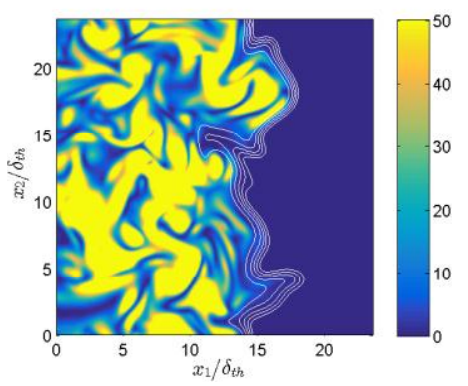

(d)

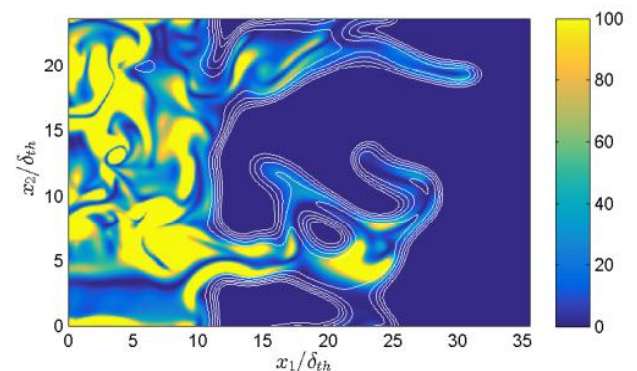

FIGURE 2. Distribution of $\Omega^{*} \times \delta_{t h}^{2} / S_{L}^{2}$ in the central $x_{1}-x_{2}$ plane when statistics have been extracted for (a) case A; (b) case B; (c) case C and (d) case G. The contours of $c$ from 0.1 to 0.9 in steps of 0.2 (left to right) are shown by white lines.

\section{1 distribution of enstrophy $\Omega$ and pseudo-enstrophy $\Omega^{*}$}

The distributions of normalised enstrophy $\Omega \times \delta_{t h}^{2} / S_{L}^{2}$ in the central $x_{1}-x_{2}$ mid-plane for selected cases are shown in Fig. 1 where the contours of $c$ from 0.1 to 0.9 (left to right) are superimposed on the enstrophy field. The corresponding distributions of 
$\Omega^{*} \times \delta_{t h}^{2} / S_{L}^{2}$ and $\left|\vec{\omega}_{\times}^{*}\right| \times \delta_{t h} / S_{L}=|\nabla \rho \times \overrightarrow{\mathrm{u}}| \times \delta_{t h} / \rho_{0} S_{L}$ are shown in Figs. 2 and 3 respectively. It is evident from Fig. 1 that high values of enstrophy $\Omega$ are obtained principally on the unburned gas side of the flame and $\Omega$ drops significantly on the burned gas side for cases B-G. This drop of enstrophy (alternatively vorticity magnitude) across the flame front is consistent with previous findings [18,21,22]. However, an opposite trend is observed in case A where $\Omega \times \delta_{t h}^{2} / S_{L}^{2}$ shows a local augmentation in magnitude across the flame which is again consistent with the observations of Treurniet et al. [21] and Lipatnikov et al. [22].

(a)

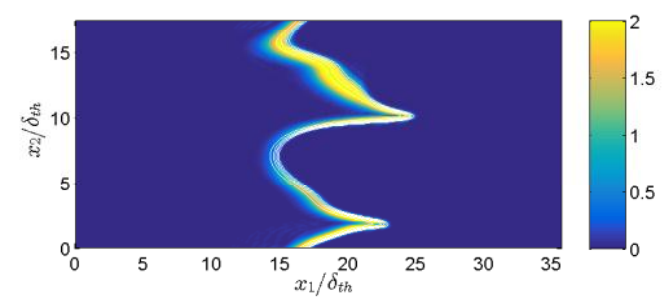

(c)

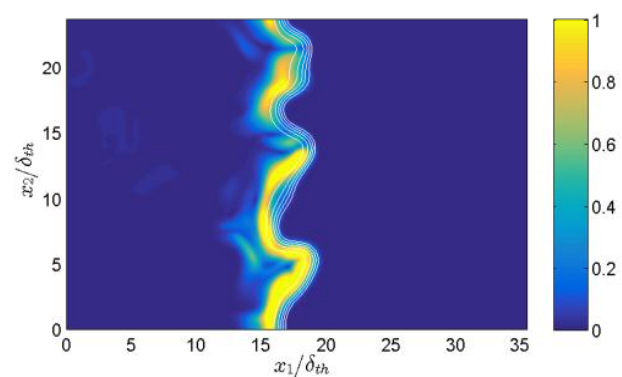

(b)

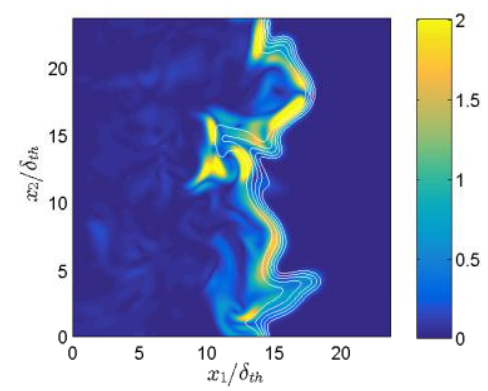

(d)

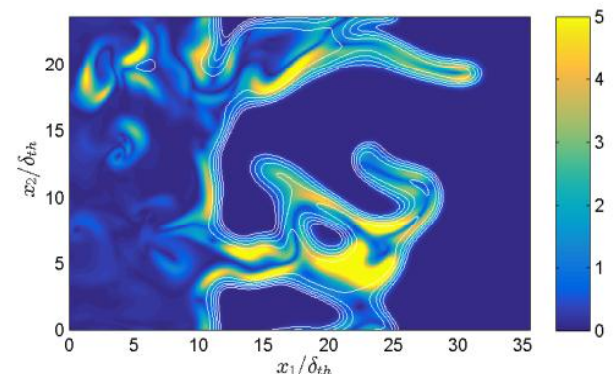

FiguRE 3. Distribution of $\left|\vec{\omega}_{\times}^{*}\right| \times \delta_{t h} / S_{L}=|\nabla \rho \times \overrightarrow{\mathrm{u}}| \times \delta_{t h} / \rho_{0} S_{L}$ in the central $x_{1}-x_{2}$ plane when statistics have been extracted for (a) case A; (b) case B; (c) case C and (d) case G. The contours of $c$ from 0.1 to 0.9 in steps of 0.2 (left to right) are shown by white lines.

The distribution of $\Omega^{*} \times \delta_{t h}^{2} / S_{L}^{2}$ in Fig. 2 reveals a sharp drop of pseudoenstrophy in the burned gas for all cases and even for case A which shows local augmentation of $\Omega \times \delta_{t h}^{2} / S_{L}^{2}$ across the flame front. It is worth nothing that density drops from unburned gas to burned gas side which contributes to a sharper drop of $\Omega^{*} \times \delta_{t h}^{2} / S_{L}^{2}$ across the flame than in the case of $\Omega \times \delta_{t h}^{2} / S_{L}^{2}$. The distribution of 
$\left|\vec{\omega}^{*}-\vec{\omega}_{\|}^{*}\right| \times \delta_{t h} / S_{L}=\left|\vec{\omega}_{\times}^{*}\right| \times \delta_{t h} / S_{L}$ indicates that this contribution remains active only within the flame front where the effects of density gradient are strong due to chemical heat release. For low Mach number unity Lewis number flames, the mixture gas density $\rho$ can be expressed as: $\rho=\rho_{0} /(1+\tau c)$ [45], which leads to $\nabla \rho=$ $-\rho^{2} \tau \nabla c / \rho_{0}$. Thus, $\left|\vec{\omega}_{\times}^{*}\right| \times \delta_{t h} / S_{L} \quad$ can be expressed as: $|\nabla \rho \times \vec{u}| \times \delta_{t h} / \rho_{0} S_{L}=$ $\tau\left|\rho^{2} \nabla c \times \vec{u}\right| \times \delta_{t h} / \rho_{0}^{2} S_{L}$. Non-zero values of $\nabla c$ exist only within the flame front and thus $\left|\vec{\omega}_{\times}^{*}\right| \times \delta_{t h} / S_{L}$ remains active only within the flame front.

(a)

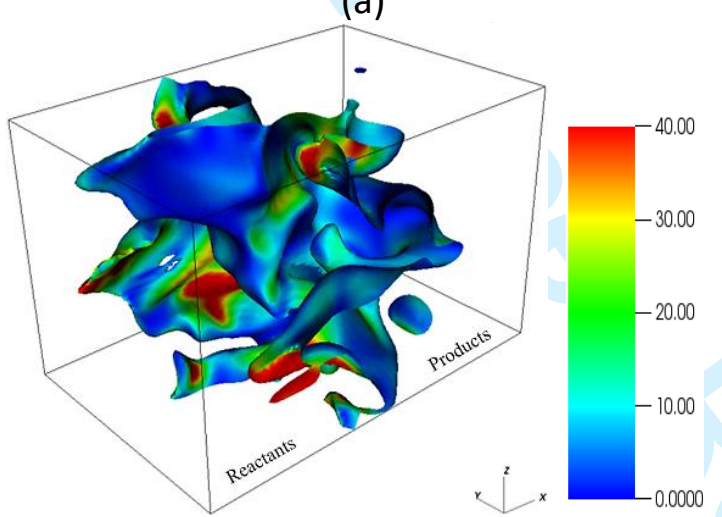

(c)

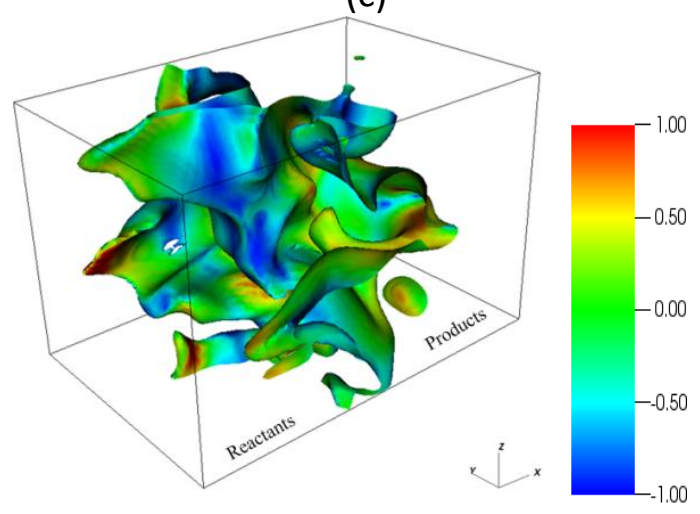

(b)

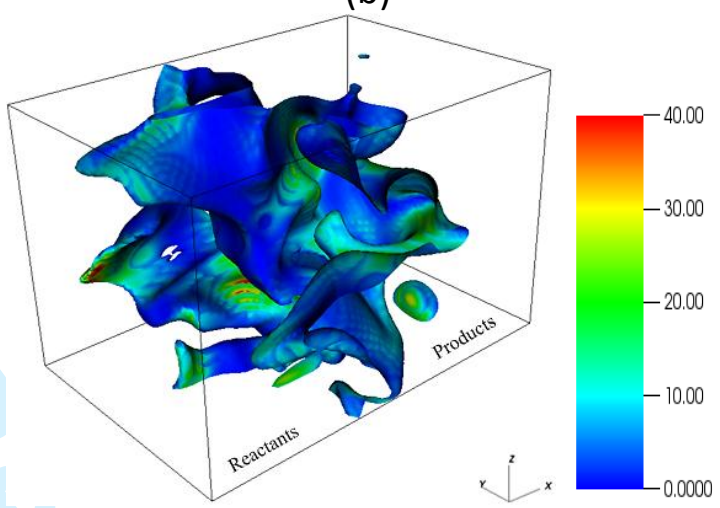

(d)

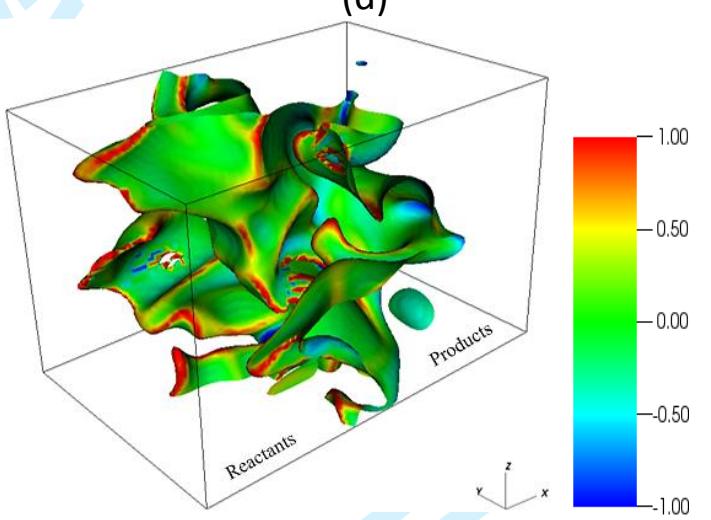

(e)

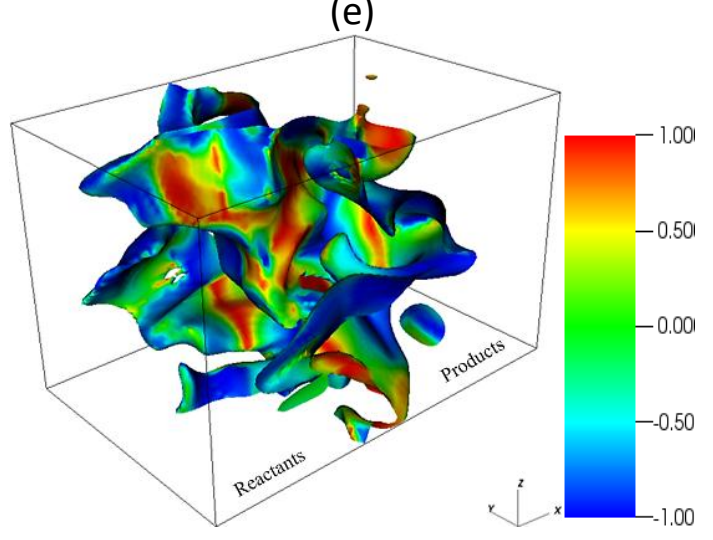


FIGURE 4. The flame surface (i.e. $c=0.8$ ) of case $\mathrm{G}$ coloured by local values of (a) $\Omega \times \delta_{t h}^{2} / S_{L}^{2}$, (b) $\Omega^{*} \times \delta_{t h}^{2} / S_{L}^{2} \quad$, (c) $\left|\vec{\omega}_{\times}^{*}\right| \times \delta_{t h} / S_{L}=|\nabla \rho \times \vec{u}| \times \delta_{t h} / \rho_{0} S_{L}$, (d) normalised curvature $\kappa_{m} \times \delta_{t h}$ and (e) $\cos \theta_{\times}=\vec{\omega}_{\times}^{*} \cdot \vec{\omega} /\left|\vec{\omega}_{\times}^{*}\right||\vec{\omega}|$.

The flame surface (i.e. $c=0.8$ ) coloured by local values of $\Omega \times \delta_{t h}^{2} / S_{L}^{2}$, $\Omega^{*} \times \delta_{t h}^{2} / S_{L}^{2},\left|\vec{\omega}_{\times}^{*}\right| \times \delta_{t h} / S_{L}$ for case $\mathrm{G}$ is shown in Fig. 4 along with the distributions of normalised curvature $\kappa_{m} \times \delta_{t h}$ and $\cos \theta_{\times}=\vec{\omega}_{\times}^{*} \cdot \vec{\omega} /\left(\left|\vec{\omega}_{\times}^{*}\right||\vec{\omega}|\right)$ on the flame surface. The curvature is defined as $\kappa_{m}=0.5 \nabla \cdot(-\nabla c /|\nabla c|)$ and according to this definition a positive (negative) curvature corresponds to the flame surface convex (concave) to the reactants, which can be confirmed from Fig. 4. It can be seen from Fig. 4 that $\Omega^{*} \times$ $\delta_{t h}^{2} / S_{L}^{2}$ assumes smaller values than $\Omega \times \delta_{t h}^{2} / S_{L}^{2}$ because of density weighting (i.e. involvement of $\rho / \rho_{0}$ in $\left.\Omega^{*}=\left(\rho \vec{\omega} / \rho_{0}+(\nabla \rho \times \vec{u}) / \rho_{0}\right) \cdot\left(\rho \vec{\omega} / \rho_{0}+(\nabla \rho \times \vec{u}) / \rho_{0}\right) / 2\right)$ and vector addition between $\rho \vec{\omega} / \rho_{0}$ and $\nabla \rho \times \vec{u} / \rho_{0}$. The distribution of $\cos \theta_{\times}$indicates that the quantities $\vec{\omega}_{\|}^{*}=\rho \vec{\omega} / \rho_{0}$ and $\vec{\omega}_{\times}^{*}=(\nabla \rho \times \vec{u}) / \rho_{0}$ are not aligned, and there is a finite probability of finding instances where $\vec{\omega}_{\times}^{*}$ acts in the opposite direction to $\vec{\omega}_{\|}^{*}$. Furthermore, Fig. 4 shows that $\Omega \times \delta_{t h}^{2} / S_{L}^{2}, \Omega^{*} \times \delta_{t h}^{2} / S_{L}^{2},\left|\vec{\omega}_{\times}^{*}\right| \times \delta_{t h} / S_{L}$ and $\cos \theta_{\times}$do not show any preferential trend with respect to local flame curvature $\kappa_{m}$.

\section{2 statistical properties of vorticity $\vec{\omega}$ and pseudo-vorticity $\vec{\omega}^{*}$}

The relative orientation of $\vec{\omega}$ and $\vec{\omega}^{*}$ as well as between these quantities with the flame surface normal and strain rate field is important to analyse the turbulent statistics. The distributions of joint PDF between $\cos \theta_{*}=\vec{\omega} \cdot \vec{\omega}^{*} /|\vec{\omega}|\left|\vec{\omega}^{*}\right|$ and normalised flame curvature $\kappa_{m} \times \delta_{t h}$ for cases $\mathrm{A}, \mathrm{B}, \mathrm{C}$ and $\mathrm{G}$ are shown in Fig. 5a. Other cases follow qualitatively the trend indicated by cases C and G, and thus are not shown here for the sake of brevity. 
Case $\mathrm{A}$ in Fig. 5a shows high probability of finding $\cos \theta_{*}=-1.0$ although a small cluster can be discerned at $\cos \theta_{*}=1.0$. By contrast, the sample points are clustered around $\cos \theta_{*}=1.0$ in case B. A similar qualitative trend has been observed for case $\mathrm{C}$ but the spread of $\cos \theta_{*}$ around 1.0 is relatively greater in case $\mathrm{C}$ than in case B. The spread of $\cos \theta_{*}$ from 1.0 to -1.0 is more uniform in case $\mathrm{C}$ than in case $\mathrm{G}$. Noting that $\rho=\rho_{0} /(1+\tau c)$, the fluctuations of $\vec{\omega}_{\|}^{*}$ and $\vec{\omega}_{\times}^{*}=(\nabla \rho \times \vec{u}) / \rho_{0}=$ $-\tau \rho^{2} \nabla \mathrm{c} \times \vec{u} / \rho_{0}^{2}$ scale as: $u^{\prime} / \lambda$ and $\tau u^{\prime} / \delta_{t h}$ respectively, where the fluctuating velocity and the length scales associated with the fluctuations of vorticity and velocity are scaled using rms turbulent velocity $u^{\prime}$ and Taylor micro-scale $\lambda$ respectively [11]. Since the Karlovitz number can also be written as $K a=\left(\delta_{t h} / \eta\right)^{2}$, the ratio of $\vec{\omega}_{\|}^{*} / \vec{\omega}_{\times}^{*}$ can be scaled as: $\rho \vec{\omega} /(\nabla \rho \times \vec{u}) \sim \delta_{t h} / \tau \lambda \sim K a^{0.5} / \tau R e_{t}^{0.25} \sim\left(u^{\prime} / S_{L}\right)^{0.5}\left(l / \delta_{t h}\right)^{-0.5} \tau^{-1}$. This suggests that the magnitude of $\vec{\omega}_{\times}^{*}$ increases in comparison to $\vec{\omega}_{\|}^{*}$ for large values of $\tau$ and $R e_{t}$ and small values of $K a$. It has been shown elsewhere $[29,46,47]$ that there is a considerable streamline divergence in case A due to Darrieus-Landau (DL) type of instability, which has a considerable probability of finding $-1<\vec{u} \cdot \nabla c /|\vec{u}||\nabla c|<1$. This leads to a considerable contribution of $\nabla \rho \times \vec{u}=-\tau \rho^{2} \nabla c \times \vec{u} / \rho_{0}$ in case A. The effects of DL instability are absent in cases B-G because the length scale ratio $l / \delta_{t h}$ is not large enough to support this [47] but the extent of flame wrinkling increases with increasing $u^{\prime} / S_{L}$, which gives rise to an increase in the number of samples corresponding to $-1<\vec{u} \cdot \nabla c /|\vec{u}||\nabla c|<1$. Thus, the magnitude of the cross-product $\nabla \rho \times \vec{u}=-\tau \rho^{2} \nabla c \times \vec{u} / \rho_{0}$ strengthens in comparison to $\rho \vec{\omega}$, and its direction can be significantly different to that of $\rho \vec{\omega}$ for the cases with high $R e_{t}$ and $\tau$, and small $K a$ (e.g. cases $\mathrm{A}$ and $\mathrm{G}$ ). This can be confirmed from Fig. 5b where the joint PDF distribution between $\cos \theta_{\times}$and normalised flame curvature $\kappa_{m} \times \delta_{t h}$ is shown for cases $\mathrm{A}, \mathrm{B}, \mathrm{C}$ and $\mathrm{G}$. It can be seen from Fig. $5 \mathrm{~b}$ that $\cos \theta_{\times}$is clustered around -1 but 
its distribution spreads from -1.0 to 1.0 in an increasingly uniform manner from case $\mathrm{C}$ to case G. Figure 5 further shows that both $\cos \theta_{\times}$and $\cos \theta_{*}$ do not show any appreciable correlation with curvature, which is consistent with the observation made from Fig. 4 . The cross product $\nabla \rho \times \vec{u}=-\tau \rho^{2} \nabla c \times \vec{u} / \rho_{0}$ is unaffected by curvature $\kappa_{m}$ for unity Lewis number flames because $\rho$ does not change on a given $c$ isosurface and $\nabla c$ is weakly related to curvature $[48-50]$.

Case

A

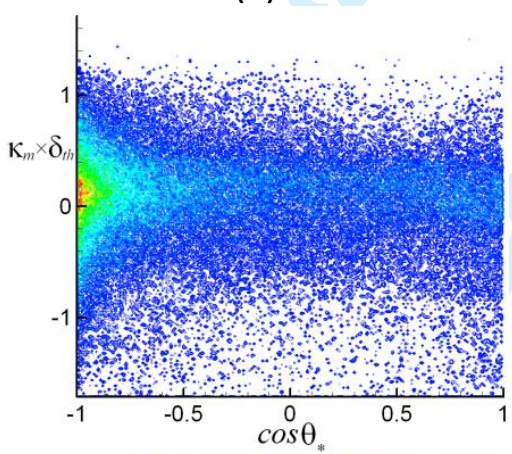

B

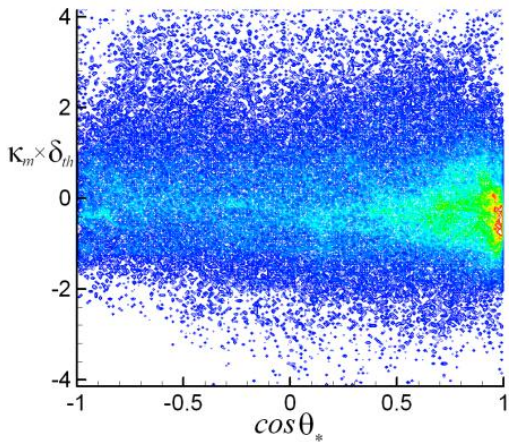

C

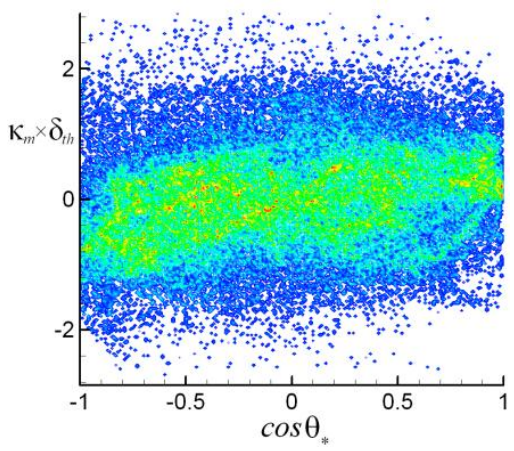

(b)
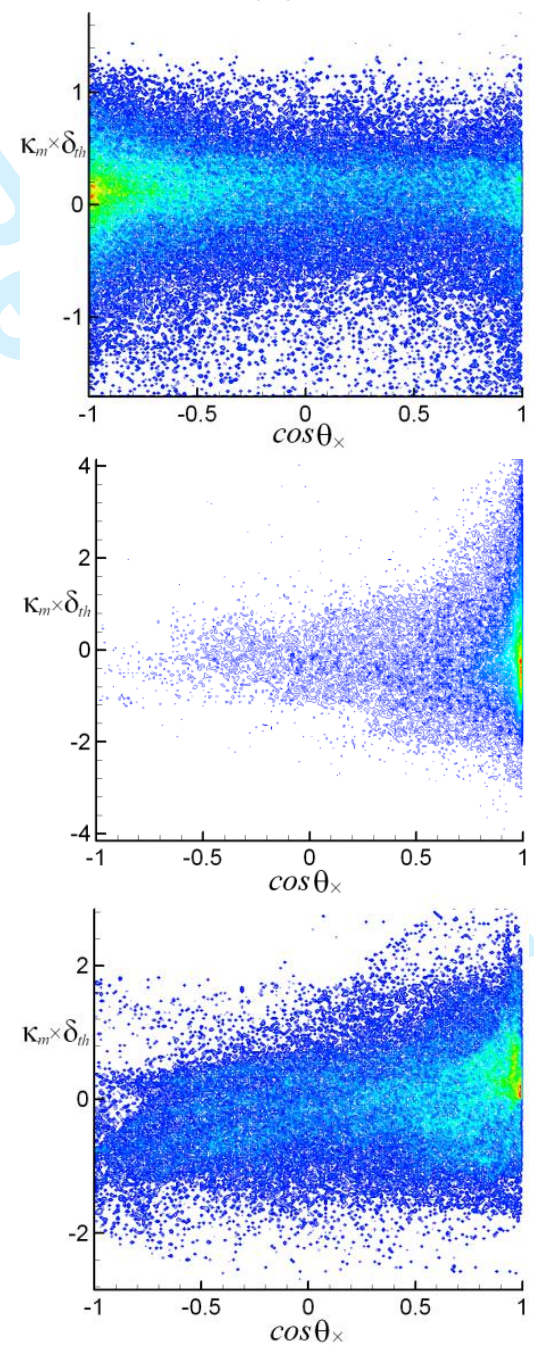


\section{G}
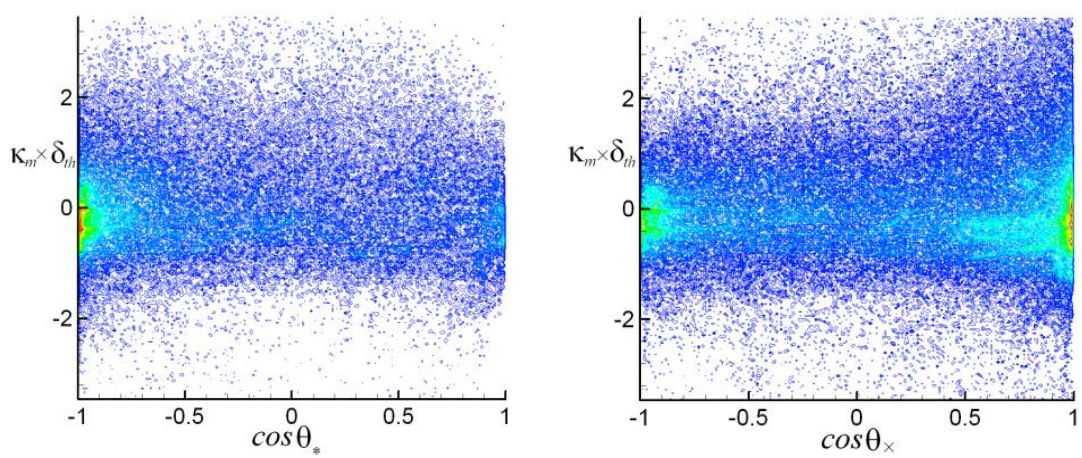

FIgURE 5. (a) Distributions of joint PDFs between $\cos \theta_{*}=\vec{\omega} \cdot \vec{\omega}^{*} /|\vec{\omega}|\left|\vec{\omega}^{*}\right|$ and normalised flame curvature $\kappa_{m} \times \delta_{t h}$ on $c=0.8$ isosurface for cases A, B, C and G; (b) Distributions of joint PDFs between $\cos \theta_{\times}=\vec{\omega}_{\times}^{*} \cdot \vec{\omega} /\left|\vec{\omega}_{\times}^{*}\right||\vec{\omega}|$ and normalised flame curvature $\kappa_{m} \times \delta_{t h}$ on $c=0.8$ isosurface for cases $\mathrm{A}, \mathrm{B}, \mathrm{C}$ and $\mathrm{G}$. The magnitude of joint PDFs increases from blue to red in this and subsequent figures.

The joint PDFs of $\cos \theta_{\times}$and $\cos \theta_{*}$ with normalised tangential strain rate $a_{T} \times \delta_{t h} / S_{L}$ (where tangential strain rate is given by: $a_{T}=\left(\delta_{i j}-N_{i} N_{j}\right) \partial u_{i} / \partial x_{j}$ with $N_{i}=-\left(\partial c / \partial x_{i}\right) /|\nabla c|$ being the $i^{\text {th }}$ flame normal component) look qualitatively very similar to the corresponding joint PDFs between $\cos \theta_{\times}$and $\cos \theta_{*}$ and normalised flame curvature $\kappa_{m} \times \delta_{t h}$ shown in Fig. 5 and thus are not shown in the paper. In particular, both $\cos \theta_{\times}$and $\cos \theta_{*}$ do not show any appreciable correlation with tangential strain rate $a_{T}$. The cross product $\nabla \rho \times \vec{u}=\tau \rho^{2} \nabla c \times \vec{u} / \rho_{0}$ is unaffected by tangential strain rate $a_{T}$ for unity Lewis number flames because $\rho$ does not change on a given $c$ isosurface, and remains independent of $a_{T}$.

For the following discussion it is useful to decompose strain rate into its most extensive, intermediate and most compressive principal strain components (i.e. $e_{\alpha}, e_{\beta}$ and $e_{\gamma}$ respectively) and to analyse their alignments with the vorticity vector $\vec{\omega}$ by looking at the angles $\theta_{\alpha}, \theta_{\beta}$ and $\theta_{\gamma}$ between $\vec{\omega}$ and $e_{\alpha}, e_{\beta}$ and $e_{\gamma}$, respectively. The corresponding angles for $\vec{\omega}^{*}$ are given by $\theta_{\alpha}^{*}, \theta_{\beta}^{*}$ and $\theta_{\gamma}^{*}$. Furthermore it is worth noting that the vortex-stretching term $T_{I}$ can be expressed as:

$$
T_{I}=\overline{2 \Omega\left(e_{\alpha} \cos ^{2} \theta_{\alpha}+e_{\beta} \cos ^{2} \theta_{\beta}+e_{\gamma} \cos ^{2} \theta_{\gamma}\right)}
$$


A high probability of $\left|\cos \theta_{i}\right|=1.0\left(\left|\cos \theta_{i}^{*}\right|=1.0\right)$ corresponds to a collinear alignment between $\vec{\omega}\left(\vec{\omega}^{*}\right)$ with the eigendirection corresponding to $e_{i}$. The probability density functions (PDFs) of $\left|\cos \theta_{\alpha}\right|,\left|\cos \theta_{\beta}\right|$ and $\left|\cos \theta_{\gamma}\right|$ for $c=0.1-0.9$ (in steps of 0.2) isosurfaces for cases A, B, C and G are shown in Fig. 6 and the corresponding PDFs for $\left|\cos \theta_{\alpha}^{*}\right|,\left|\cos \theta_{\beta}^{*}\right|$ and $\left|\cos \theta_{\gamma}^{*}\right|$ are shown in Fig. 7.

Case

A

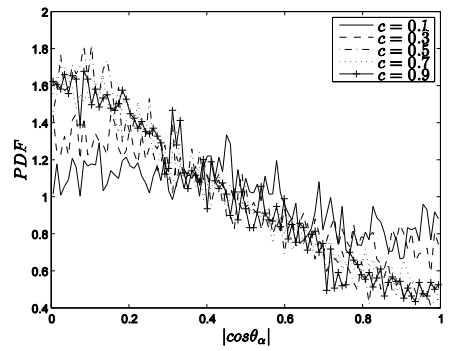

B

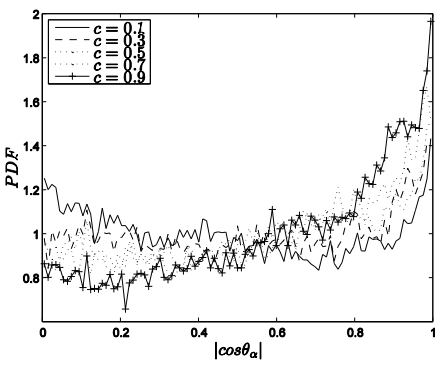

C

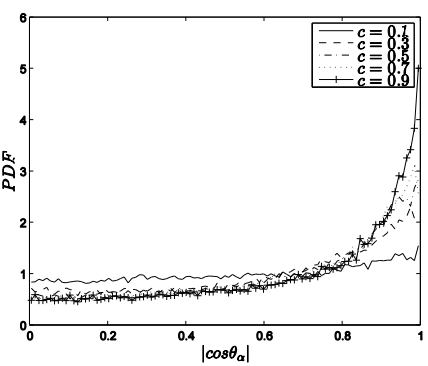

(b)
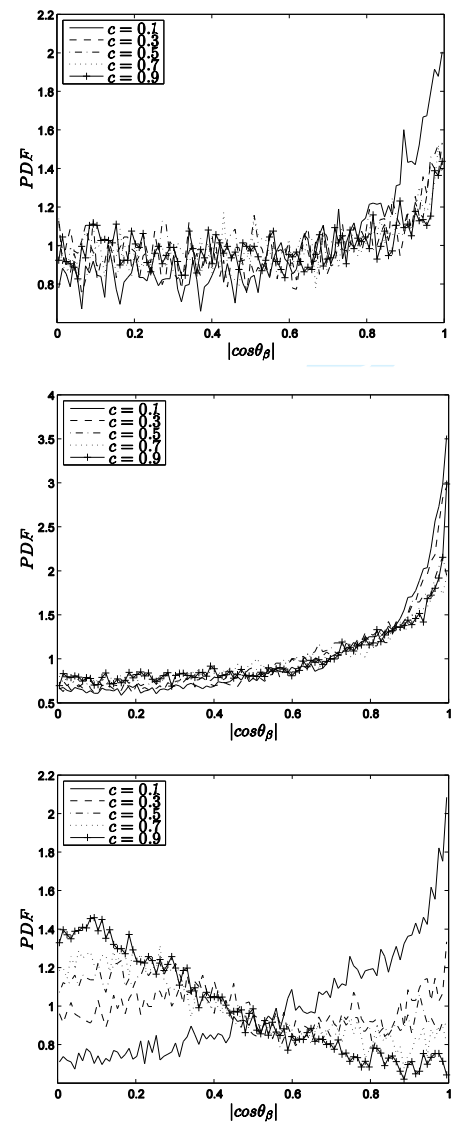

(c)
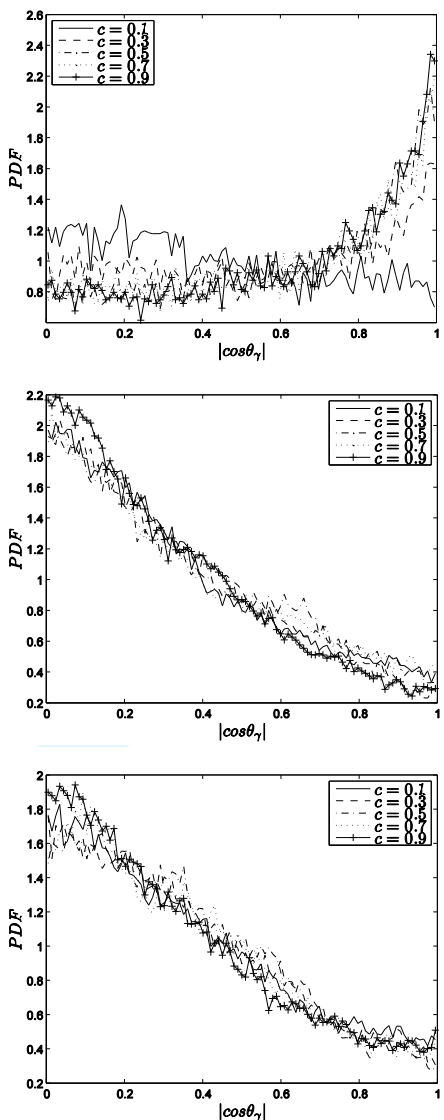
G

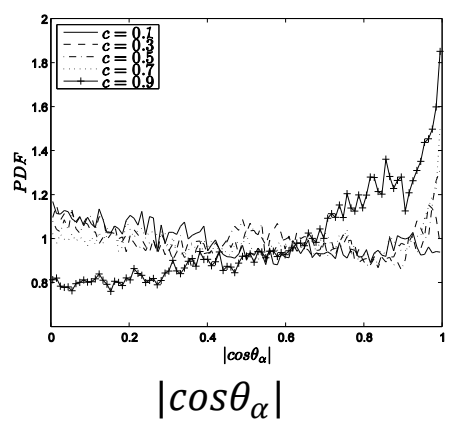

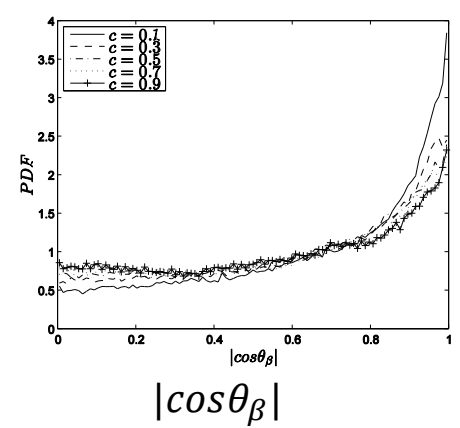

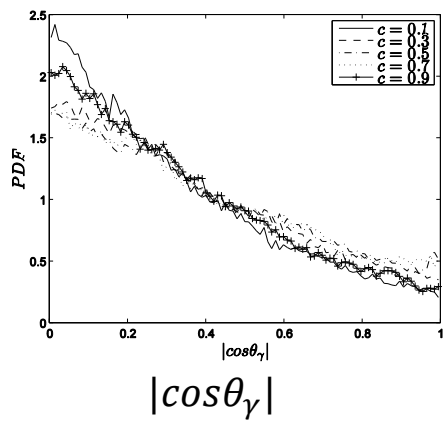

FIGURE 6. (a) The probability density functions (PDFs) of $\left|\cos \theta_{\alpha}\right|$ for $c=0.1-0.9$ (in steps of 0.2) isosurfaces for cases A, B, C and G. (b) The probability density functions (PDFs) of $\left|\cos \theta_{\beta}\right|$ for $c=0.1-0.9$ (in steps of 0.2 ) isosurfaces for cases $\mathrm{A}, \mathrm{B}, \mathrm{C}$ and G. (c) The probability density functions (PDFs) of $\left|\cos \theta_{\gamma}\right|$ for $c=0.1-0.9$ (in steps of 0.2) isosurfaces for cases $\mathrm{A}, \mathrm{B}, \mathrm{C}$ and $\mathrm{G}$.

Case

(a)

A

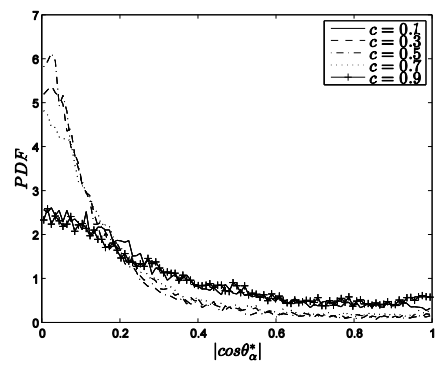

B

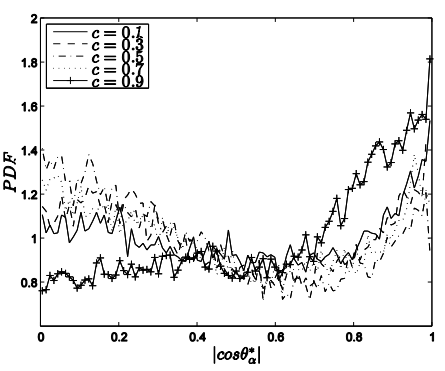

(b)
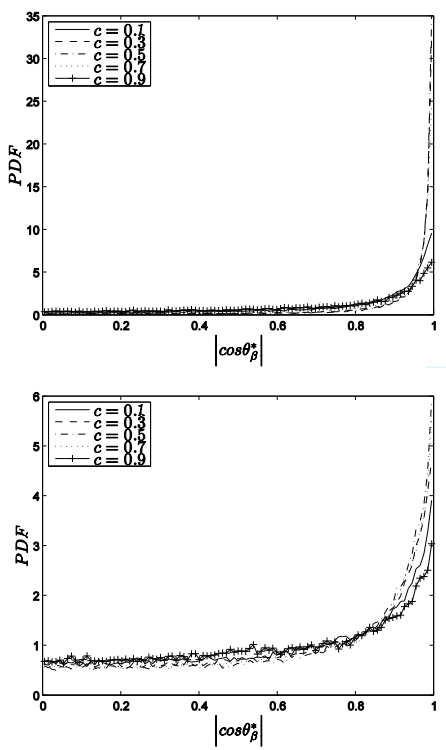

(c)
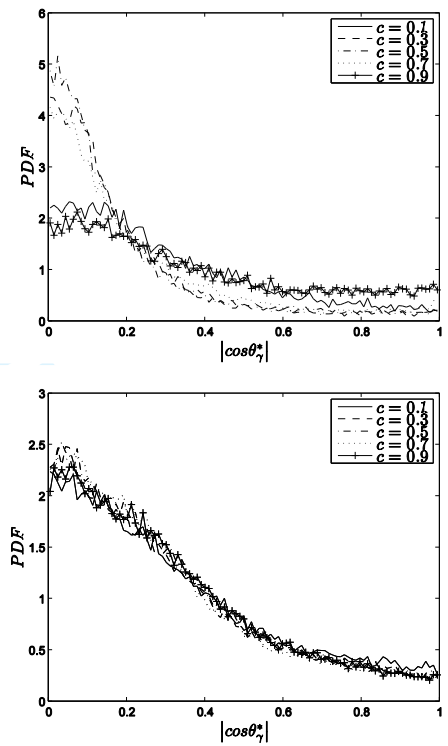
$\mathrm{C}$

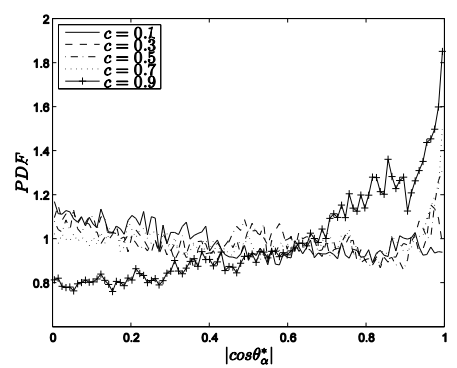

G

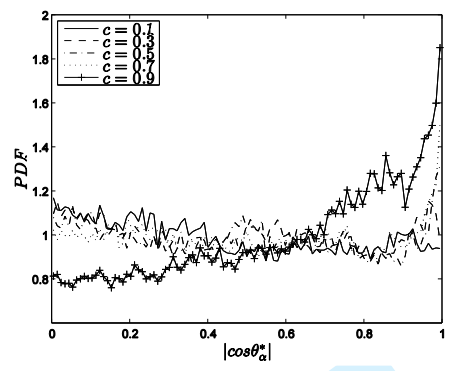

$\left|\cos \theta_{\alpha}^{*}\right|$
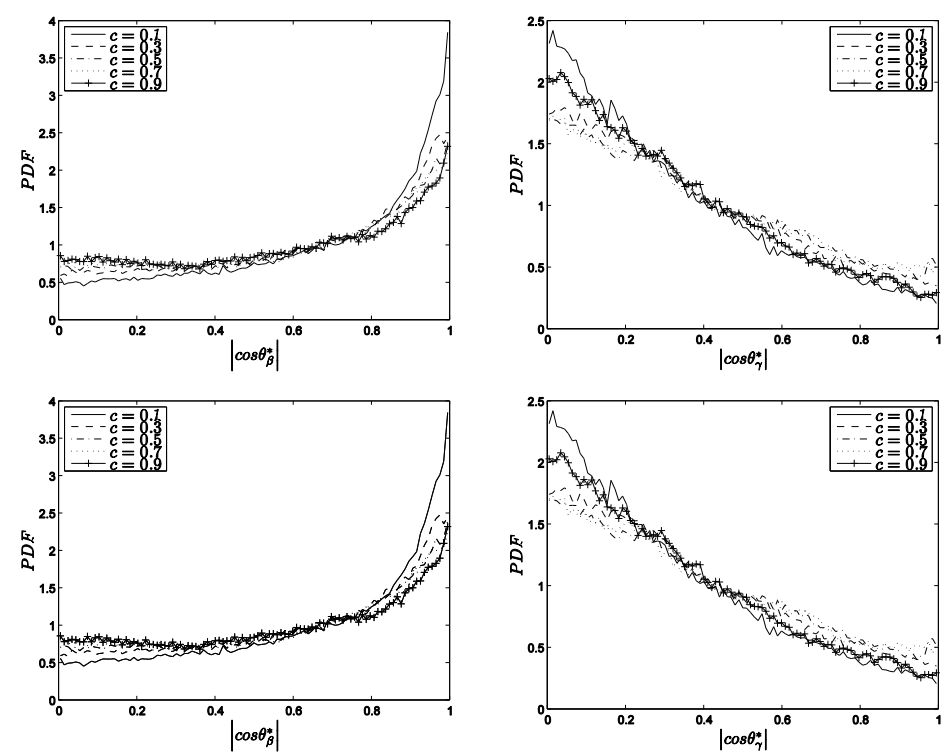

$\left|\cos \theta_{\beta}^{*}\right|$

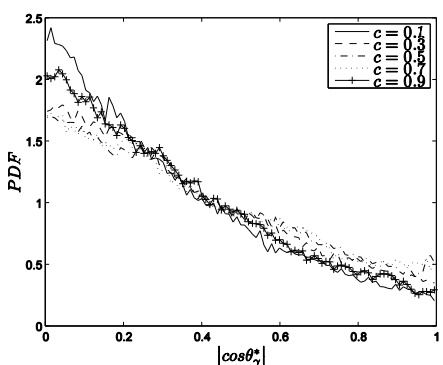

$\left|\cos \theta_{\gamma}^{*}\right|$

FIGURE 7. (a) The probability density functions (PDFs) of $\left|\cos \theta_{\alpha}^{*}\right|$ for $c=0.1-0.9$ (in steps of 0.2) isosurfaces for cases A, B, C and G. (b) The probability density functions (PDFs) of $\left|\cos \theta_{\beta}^{*}\right|$ for $c=0.1-0.9$ (in steps of 0.2 ) isosurfaces for cases A, B, C and G. (c) The probability density functions (PDFs) of $\left|\cos \theta_{\gamma}^{*}\right|$ for $c=0.1-0.9$ (in steps of 0.2) isosurfaces for cases A, B, C and G.

It is worth noting (refer to Eq. 9), that the vortex-stretching term $T_{I}$ depends only on the magnitude of $\cos \theta_{i}$ but not on their sign. Therefore the norm of $\cos \theta_{i}$ and $\cos \theta_{i}{ }^{*}$ is considered in Figs. 6 and 7 respectively. It is evident from Fig. 6 that $\vec{\omega}$ predominantly aligns with intermediate principal strain rate $e_{\beta}$ for cases $\mathrm{A}, \mathrm{B}$ and $\mathrm{G}$ but the alignment with the most extensive principal strain rate $e_{\alpha}$ increases in the reaction zone in cases $\mathrm{B}$ and $\mathrm{G}$, whereas $\vec{\omega}$ aligns predominantly with $e_{\beta}$ and $e_{\gamma}$ and not with $e_{\alpha}$ in case A. This behaviour is consistent with previous findings [20] and interested readers are referred to Chakraborty [20] for the physical explanations for this alignment. It has been found that $\vec{\omega}$ aligns predominantly with $e_{\alpha}$ in cases $\mathrm{C}$ and $\mathrm{D}$, and the alignments of $\vec{\omega}$ with $e_{\beta}$ and $e_{\gamma}$ decrease from the unburned to burned gas side of the flame. The net contribution of vortex stretching and dilatation terms (i.e. $\vec{\omega} \cdot \nabla \vec{u}-$ $\vec{\omega} \nabla \cdot \vec{u})$ has the following components $|\vec{\omega}|\left(e_{\alpha}-\nabla \cdot \vec{u}\right) \cos \theta_{\alpha},|\vec{\omega}|\left(e_{\beta}-\nabla \cdot \vec{u}\right) \cos \theta_{\beta}$ and $|\vec{\omega}|\left(e_{\gamma}-\nabla \cdot \vec{u}\right) \cos \theta_{\gamma}$ in the eigendirections corresponding to $e_{\alpha}, e_{\beta}$ and $e_{\gamma}$ respectively. 


\section{Case}

(a)

\section{A}

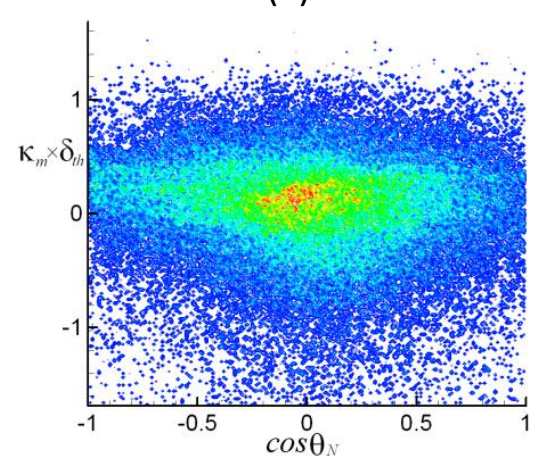

(b)

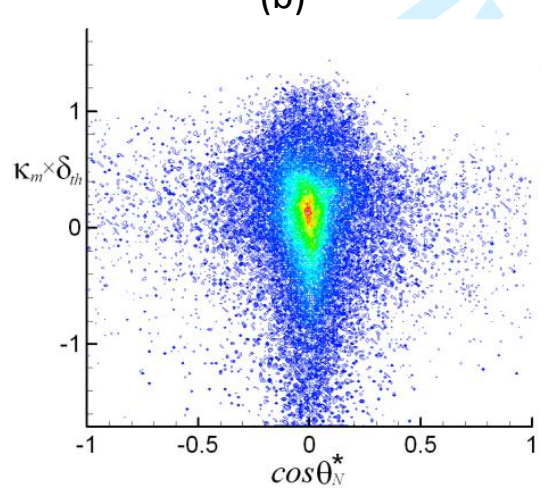


B
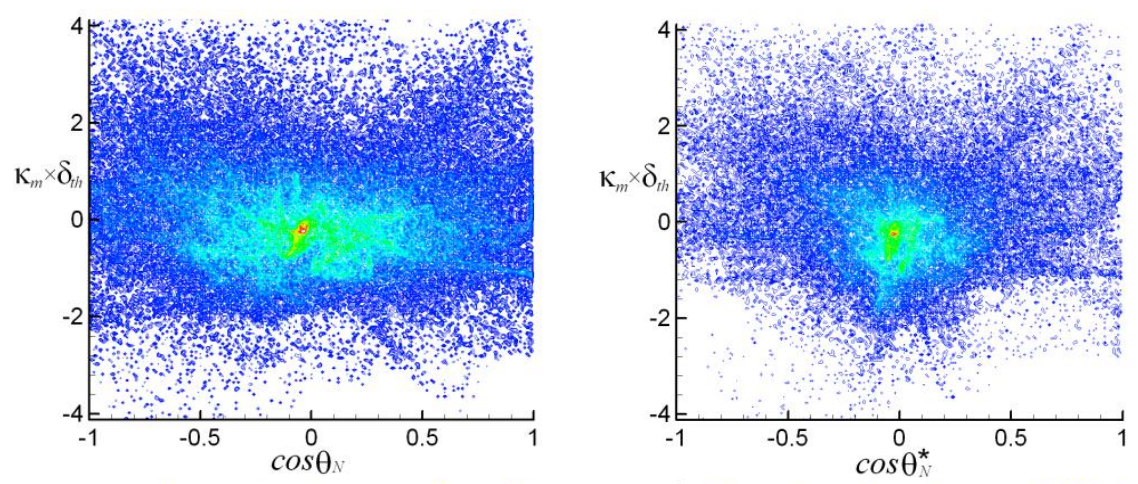

C
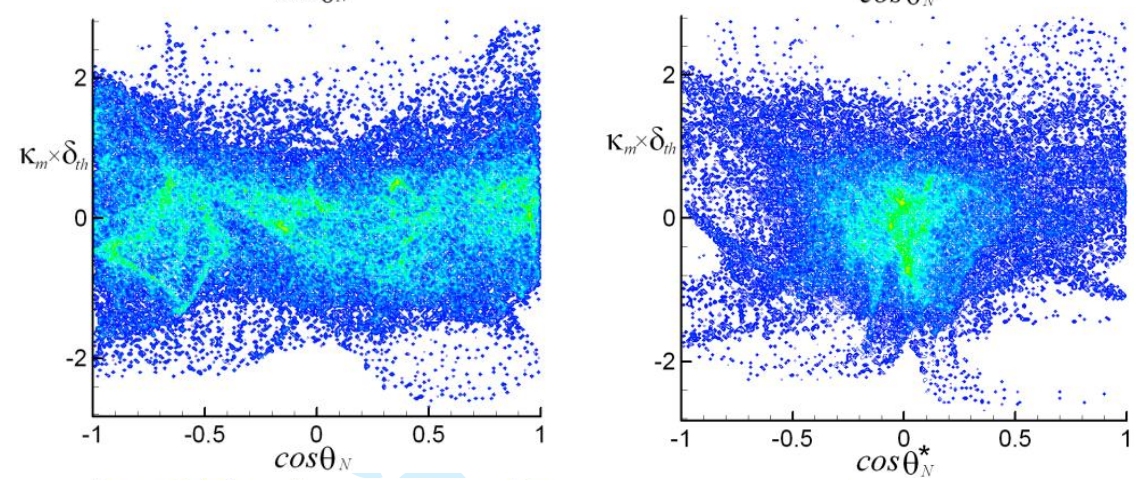

G
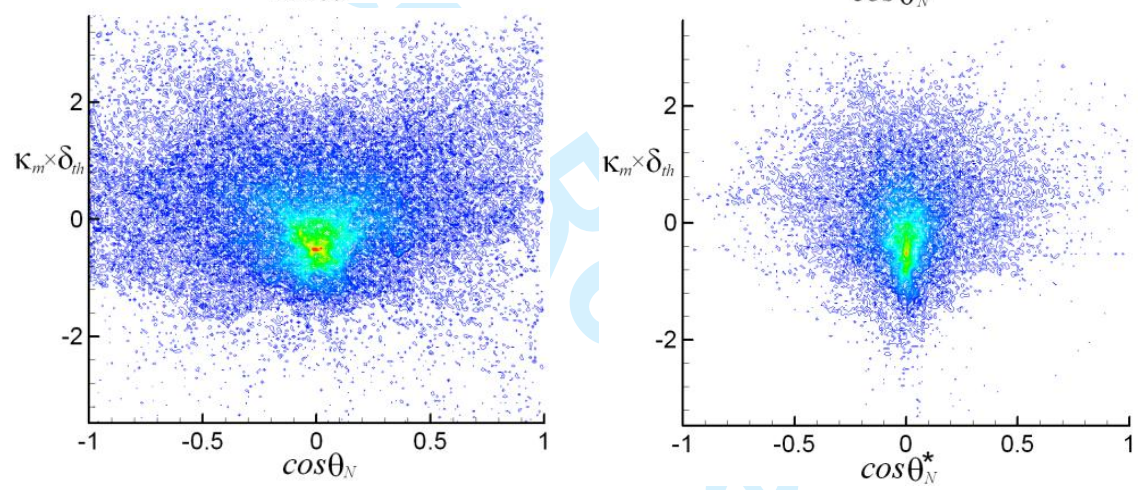

FIGURE 8. (a) Distributions of joint PDFs between $\cos \theta_{N}=-\vec{\omega} \cdot \vec{N} /(|\vec{\omega}| \cdot|\vec{N}|)$ and normalised flame curvature $\kappa_{m} \times \delta_{t h}$ on $c=0.8$ isosurface for cases A, B, C and G; (b) Distributions of joint PDFs between $\cos \theta_{N}^{*}=-\vec{\omega}^{*} \cdot \vec{N} /\left(\left|\vec{\omega}^{*}\right| \cdot|\vec{N}|\right)$ and normalised flame curvature $\kappa_{m} \times \delta_{t h}$ on $c=0.8$ isosurface for cases $\mathrm{A}, \mathrm{B}, \mathrm{C}$ and $\mathrm{G}$.

The distributions of joint PDF between $\cos \theta_{N}=-\vec{\omega} \cdot \vec{N} /(|\vec{\omega}| \cdot|\vec{N}|)$ and flame curvature $\kappa_{m}$ for cases A, B, C and G are shown in Fig. 8a and the joint PDFs between $\cos \theta_{N}^{*}=-\vec{\omega}^{*} \cdot \vec{N} /\left(\left|\vec{\omega}^{*}\right| \cdot|\vec{N}|\right)$ and flame curvature $\kappa_{m}$ for the same cases are shown in Fig. 8b. Evidently, it is possible to locally obtain high magnitude of the negative curvature in case A, which arises due to sharply curved cusps between positively curved bulges due to Darrieus-Landau (DL) type of instability in this flame [56]. Such a tendency is not observed for other cases because the values of $l / \delta_{t h}$ in these cases are 
not large enough to support the DL instability. Moreover, for case A and case C, the conditional mean curvature is almost independent of the value of $\cos \theta_{N}$, while case $\mathrm{G}$ shows a clear dependence, i.e. the mean curvature is smaller at $\cos \theta_{N}=0$ and larger at $\cos \theta_{N}= \pm 1$. In case $\mathrm{A}$, the dilatation rate remains almost equal to the extensive principal strain rate [20], and thus the enstrophy is selectively suppressed in the direction of the most extensive principal strain rate throughout the flame [20] due to the strong action of dilatation rate (see the term $\vec{t}_{3}$ on right hand side of eq. 2 ). Thus, the vorticity vector $\vec{\omega}$ in case $\mathrm{A}$ is aligned with the intermediate $e_{\beta}$ and the most compressive $e_{\gamma}$ principal strain rates irrespective of the curvature values, whereas $\vec{N}$ aligns predominantly with the most extensive principal strain rate $e_{\alpha}$ in this case [56]. The orthogonality of principal strain directions leads to a high probability of obtaining orthogonality between $\vec{\omega}$ and $\vec{N}$, which is reflected in high probability of finding $\cos \theta_{N}=0$ in case A. As $\vec{\omega}$ in cases B, E-G aligns with the intermediate strain rate $e_{\beta}$ irrespective of the curvature values, whereas $\vec{N}$ aligns predominantly with the most extensive principal strain rate $e_{\alpha}$ in the reaction zone (e.g. $c=0.8$ isosurface), this leads to high probability of finding $\cos \theta_{N}=0$ in these cases. It has already been shown in Fig. 6 that $\vec{\omega}$ aligns preferentially with the eigenvector corresponding to $e_{\alpha}$ and $e_{\beta}$ in case $\mathrm{C}$, whereas in this case $\vec{N}$ predominantly aligns with $e_{\alpha}$. Thus, there is some degree of alignment between $\vec{\omega}$ and $\vec{N}$ in case C (also in case D), which leads to a uniform distribution between $\cos \theta_{N}=-1$ and $\cos \theta_{N}=1$.The flame normal vector $\vec{N}$ shows an increased alignment with the most extensive principal strain rate in the high dilatation rate zone (e.g. reaction zone) in $D a<1$ flames with $u^{\prime} \gg S_{L}$ [57,58]. The probability of obtaining high values of dilatation rate and the alignment of $\vec{N}$ with the most extensive principal strain rate increase with decreasing curvature [57,58]. This 
tendency increases with decreasing curvature (mainly for negatively curved zones) in $D a<1$ flames with $u^{\prime} \gg S_{L}$, and thus the conditional mean curvature at $\cos \theta_{N}=0$ shows a smaller value than those at $\cos \theta_{N}= \pm 1$ in case G. It is shown in Fig. 7 that $\vec{\omega}^{*}$ aligns preferentially with intermediate principal strain rate $e_{\beta}$, whereas $\vec{N}$ predominantly aligns with $e_{\alpha}$ on $c=0.8$ isosurface for all cases. This leads to strong clustering of points at $\cos \theta_{N}^{*}=0$ for all cases due to orthogonality of principal strain directions leading to a high probability of orthogonality between $\vec{\omega}^{*}$ and $\vec{N}$.

\subsection{Statistical nature of enstrophy $\Omega$ and pseudo-enstrophy $\Omega^{*}$ transport}

The variations of the normalised values of the terms on the right hand side of $\bar{\Omega}$ transport equation (i.e. $T_{I}, T_{I I}, T_{I I I}, T_{I V}$ and $T_{V}$ ) with $\tilde{c}$ are shown in Fig. 9. It can be seen that the mean contribution of vortex-stretching term $T_{I}$ remains positive throughout the flame brush for all cases. It was previously shown by Chakraborty [20] that the term $T_{I}$ acts to generate enstrophy for all cases irrespective of the nature of the alignment between $\vec{\omega}$ and the principal strain rates. Interested readers are referred to Chakraborty [20] for further information in this regard. The correlation between density variation and viscous action $T_{I I}$ remains small in magnitude in comparison to the other terms in cases B-G. The viscous dissipation term $T_{I I I}$ acts as a major sink term for all cases, whereas the dilatation rate term $T_{I V}$ assumes non-zero negative values only within the flame brush. However, the magnitude of the dilatation rate term $T_{I V}$ remains small in comparison to the viscous dissipation term $T_{I I I}$ for cases B-G. The baroclinic torque term $T_{V}$ generates enstrophy within the flame brush but vanishes both in the unburned and burned gas sides and its magnitude also remains small in comparison to those of $T_{I}$ and $T_{I I I}$ in cases B-G. The magnitudes of the terms $T_{I I}, T_{I V}$ and $T_{V}$ remain comparable to those of $T_{I}$ and $T_{I I I}$ in case A. In case A, $\vec{\omega}$ does not align with the most extensive 
principal strain rate [20], and thus the relative magnitude of the term $T_{I}$ remains small in comparison to $T_{I I I}$ in case A.

(a)

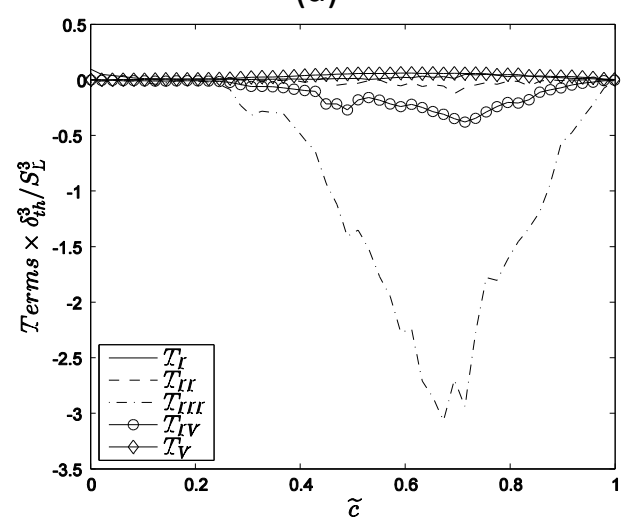

(c)

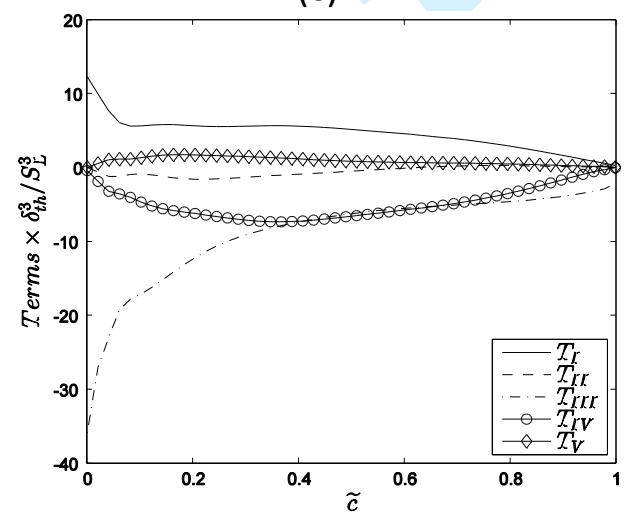

(b)

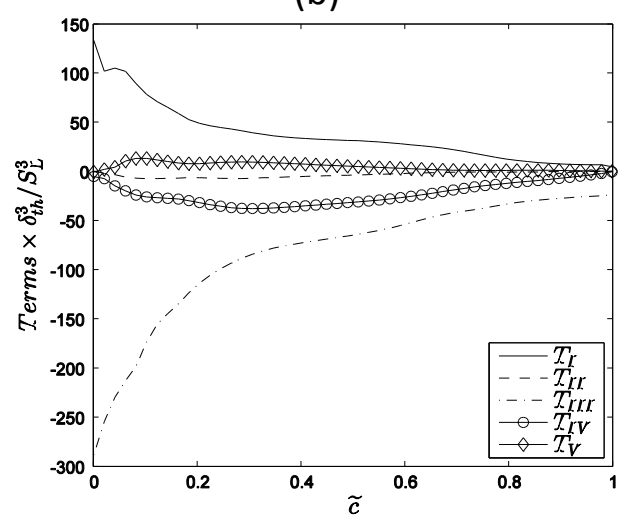

(d)

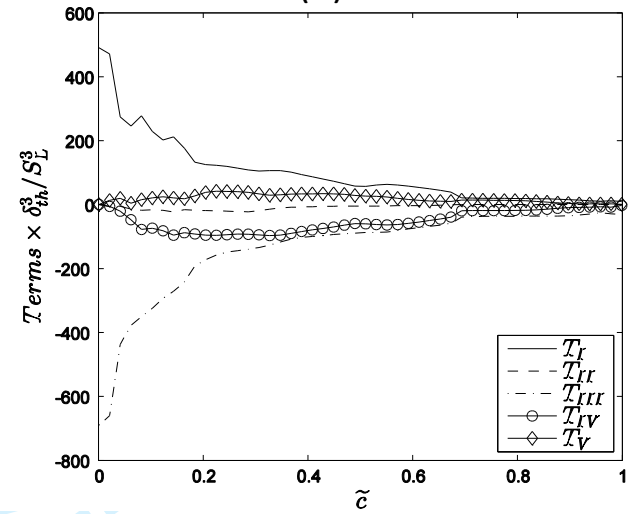

FIGURE 9. Variations of the normalised values of the terms $T_{i} \times \delta_{t h}^{3} / S_{L}^{3}$ (where $T_{i}=$ $T_{I}, T_{I I}, T_{I I I}, T_{I V}$ and $T_{V}$ ) on the right hand side of $\bar{\Omega}$ transport equation (i.e. eq. 7) with $\tilde{c}$ for (a) case A; (b) case B; (c) case C and (d) case G

(a)

(b) 


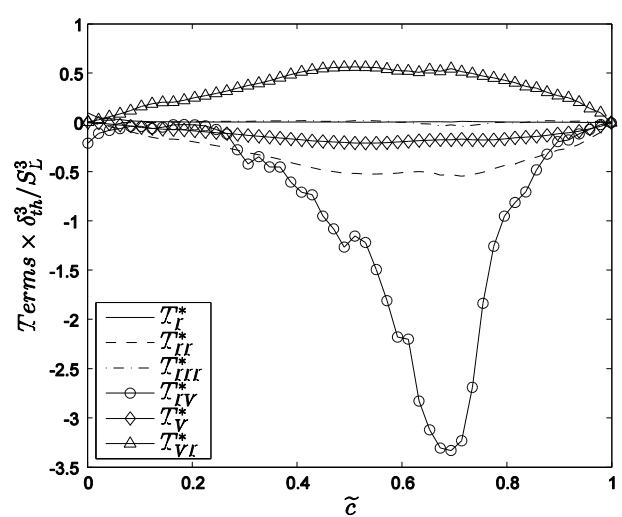

(c)

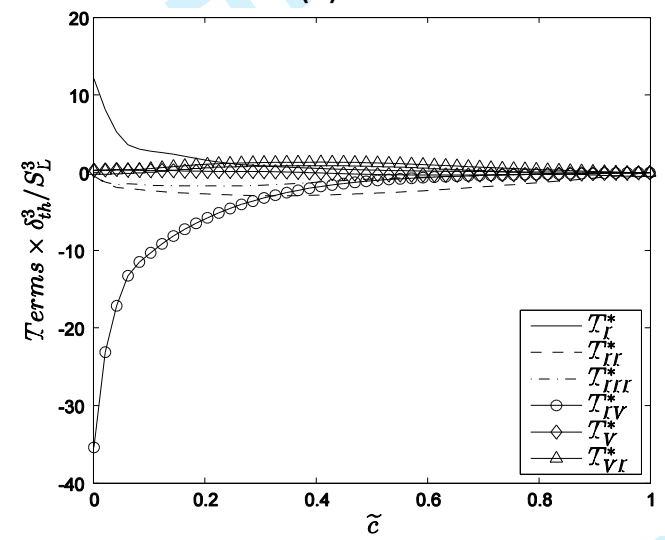

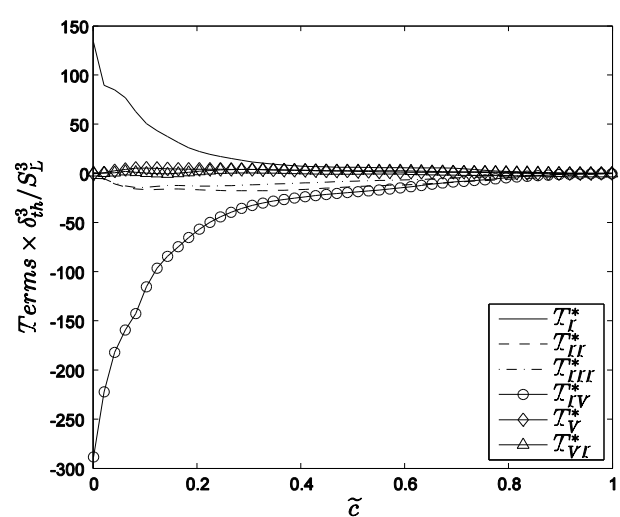

(d)

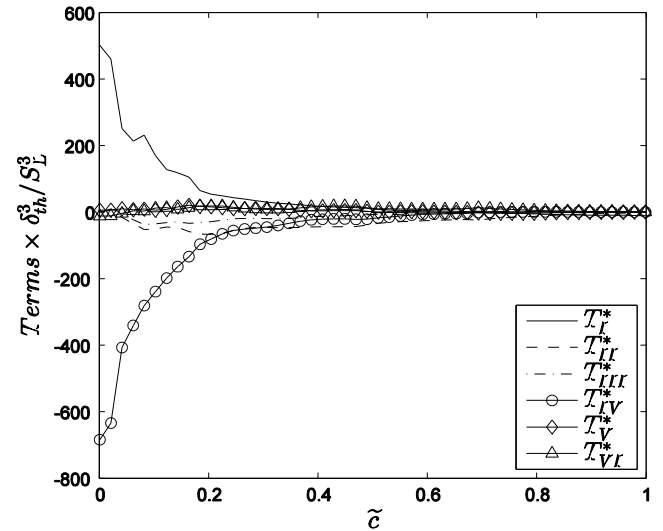

FIGURE 10. Variations of the normalised values of the terms $T_{i}^{*} \times \delta_{t h}^{3} / S_{L}^{3}$ (where $T_{i}^{*}=T_{I}^{*}-T_{V}^{*}$ and $T_{V I}^{*}$ ) on the right hand side of $\overline{\Omega^{*}}$ transport equation (i.e. eq. 8) with $\tilde{c}$ for (a) case A; (b) case B; (c) case $\mathrm{C}$ and (d) case $\mathrm{G}$.

The variations of the normalised values of the terms on the right hand side of $\overline{\Omega^{*}}$ transport equation (i.e. $T_{I}^{*}-T_{V}^{*}$ and $T_{V I}^{*}$ ) with $\tilde{c}$ are shown in Fig. 10. It can be seen from Fig. 10 that the vortex-stretching contribution $T_{I}^{*}$ acts as a major source term, whereas the viscous dissipation $T_{I V}^{*}$ acts as a leading order sink for cases B-G. The terms $T_{I I}^{*}$ and $T_{I I I}^{*}$ act as sink terms for all cases. The contributions of $T_{V}^{*}$ and $T_{V I}^{*}$ remain negligible in comparison to the magnitudes of $T_{I}^{*}$ and $T_{I V}^{*}$ for cases B-G. However, the terms $T_{V}^{*}$ and $T_{V I}^{*}$ play an important role in case $\mathrm{A}$, and the terms $T_{V I}^{*}$ and $T_{V}^{*}$ act as leading order source and sink respectively in this case. The magnitudes of $T_{I}^{*}$ and $T_{I I I}^{*}$ remain negligible in comparison to the other terms on the right hand side of $\bar{\Omega}^{*}$ transport equation in case $\mathrm{A}$. 


\section{Non-local statistics of vorticity line segments}

\subsection{Structure definition}

The nonlocal statistics aim to address the field properties and the flow topology at finite scales. Especially we define here the vorticity line segment (VLS) structure. At any spatial point in the $\vec{\omega}$ field, its corresponding vorticity line is the curve, which is locally tangential to $\vec{\omega}$. Differently from the nonreactive turbulence case where the statistics are spatially homogeneous, in reactive turbulence the vorticity line across the flame surface is of special interest to understand the flame-turbulence interaction. As shown in Fig. 11, one can find a local maximum of the vorticity magnitude $\omega=|\vec{\omega}|$ (in red) and a local minimum (in blue) by moving along the vorticity line towards two opposite directions starting from any flame surface point. The part of the vorticity line bounded by the two adjacent extremal points is defined as a VLS. This concept was first put forward by Wang [28] in studying the turbulent velocity vector field. Afterwards applications in different situations have been developed, such as nonreactive turbulent vorticity field [14] and reactive turbulence [29,30].

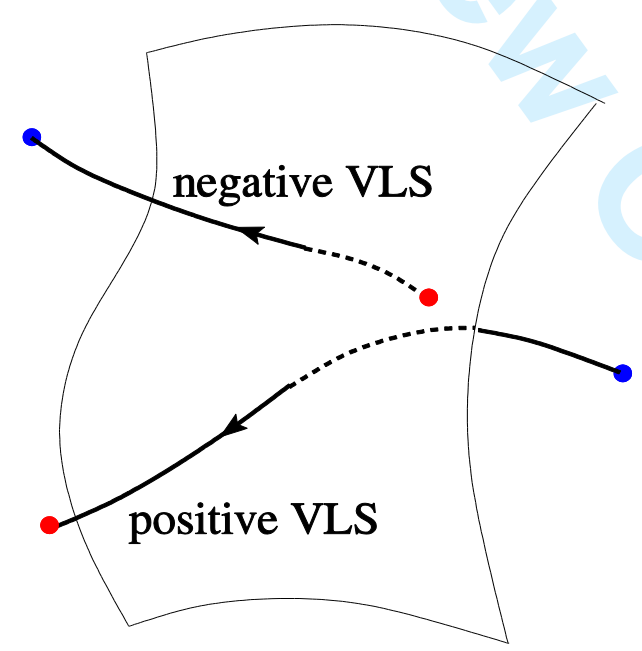

FIGURE 11. Schematic of vorticity line segment across the flame surface. According to the sign of $\Delta \omega\left(\Delta \omega^{*}\right)$ the segments can be either positive or negative. Red and blue colours indicate local maximum and local minimum respectively. 
For isothermal flows, the dynamics of extremal points is primarily determined by two different mechanisms. On the one hand turbulent convection disturbs the flow field to generate new extremal points; on the other hand, molecular diffusion acts to annihilate these extrema. Additionally, in turbulent premixed flames heat release tends to produce large segment length.

VLS can be characterized by the following two important parameters. One is the vorticity line arc length $L$ between the two extremal points; the other is $\Delta \omega$, the difference of $\omega$ along the vector direction. According to the sign of $\Delta \omega$, VLS can either be positive $(\Delta \omega>0)$ or negative $(\Delta \omega<0)$. Alternatively, VLS can also be defined based on the $\vec{\omega}^{*}$ field with the corresponding characteristic parameters $\Delta \omega^{*}$ and the arc length $L^{*}$ along $\vec{\omega}^{*}$.

(a)

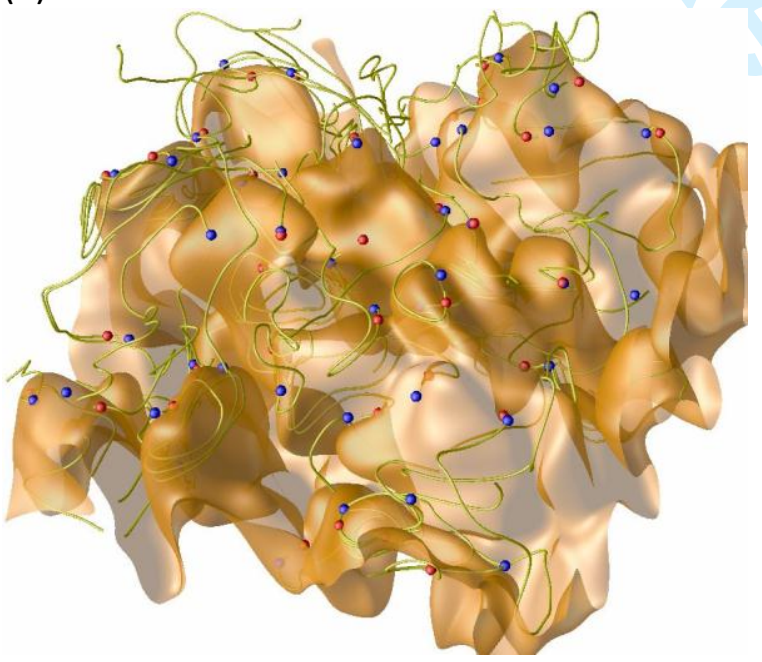

(b)

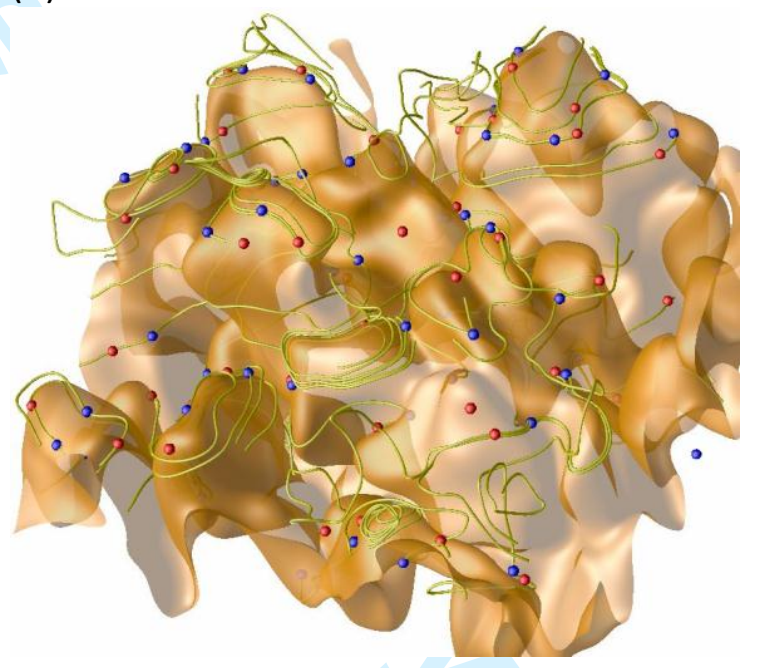

FIGURE 12. Illustration of the VLS structure across the flame front (for case A) with the local maximal (red) and minimal (blue) points for (a) $\vec{\omega}$ and (b) $\vec{\omega}^{*}$.

Examples of VLS for case A identified from DNS data processing are shown in Fig. 12 (a) and (b) for the vorticity and pseudo-vorticity case, respectively. Visually it is clear that the vorticity lines (yellow) are inclined to be locally tangential to the flame 
front, which conforms with the previous local description. The two parts of a VLS on different sides of the flame front can be very different. The natural topology of the vorticity field in the vicinity of the flame front can be represented by VLS. In principle the overall vorticity field statistics can be derived from integration of the knowledge of all the individual VLS. More importantly the definition of VLS makes it possible to describe the vorticity topology at fine scales in a quantitative manner.

\subsection{Characteristic parameter analysis}

The arc length of VLS can be understood as the characteristic scale of the local flow field. The joint PDF between $L$ and $\cos \theta_{N}$ indicates how the local fine scale depends on the relative orientation between flame normal and vorticity, which is shown for cases A, $\mathrm{B}, \mathrm{C}$ and $\mathrm{G}$ in Fig. 13. It is evident that in case $\mathrm{A}$ there is a hump in the joint PDF, i.e. the sample points with large $L$ values are concentrated around $\cos \theta_{N}=0$. The thermal expansion effects are dominant in case A due to small values of $u^{\prime}$ and it was shown elsewhere $[32,43]$ that the flame normal vector in the flames with high Damköhler number $(D a \gg 1)$ preferentially aligns locally with the most extensive principal strain rate, whereas the vorticity vector shows predominant alignment with the intermediate and the most compressive principal strain rates [20]. As the principal strain rate directions are mutually perpendicular, $\cos \theta_{N}$ is most likely to assume a zero value in case A. The locations associated with $\cos \theta_{N}=0$ in case $\mathrm{A}$ are associated regions of strong thermal expansion and this predominance of thermal expansion acts to increase the arc length $L$, which gives rise to large values of $L$ at $\cos \theta_{N}=0$ in case A. Although $\vec{\omega}$ predominantly aligns with the intermediate principal strain rate in cases B-G, the vorticity vector in these cases also shows a reasonable extent of alignment with the most extensive principal strain rate in the reaction zone (see Fig. 6), where the flame normal vector also tends to align with the most extensive principal strain rate [56-58]. Thus, $\vec{\omega}$ 
and $\vec{N}$ show a certain degree of collinear alignment in case $\mathrm{C}$, which results in a homogeneous joint PDF between $L$ and $\cos \theta_{N}$, as shown in Fig. 13 (c).

(a)

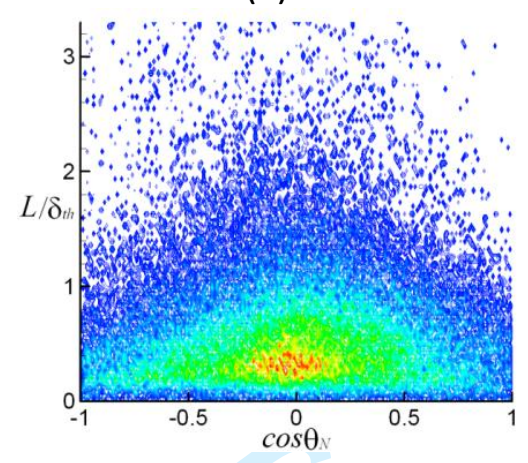

(c)

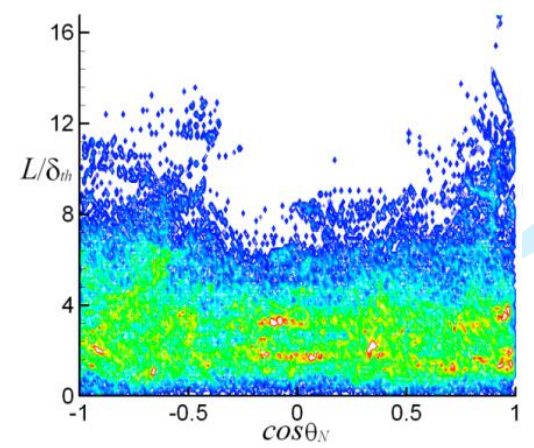

(b)

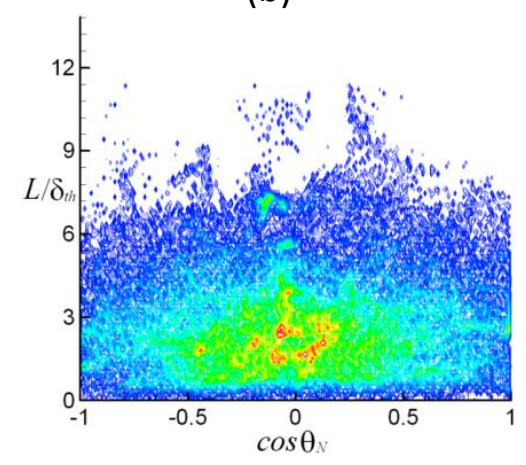

(d)

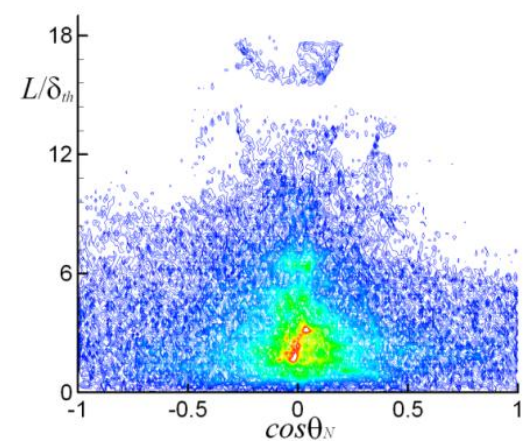

FiguRE 13. Joint PDFs between $L$ and $\cos \theta_{N}$ for $c=0.8$ in cases (a) A, (b) B, (c) C and (d) G.

Moreover the length of VLS assumes high values at $\cos \theta_{N}= \pm 1$ for case $\mathrm{C}$ under the action of strong thermal expansion because in this case the effects of thermal expansion are strong in the regions corresponding to $\cos \theta_{N}= \pm 1$ where both vorticity and the flame normal vector show collinear alignment with the most extensive principal strain rate. The flames with $u^{\prime} / S_{L} \gg 1$ (e.g. cases $F$ and G) in the TRZ regime show attributes of non-reacting turbulent flows where $\vec{\omega}$ tends to align with the intermediate principal strain rate, whereas $\vec{N}$ predominantly aligns with the most compressive principal strain rate but this alignment changes to the most extensive principal strain rate in the reaction zone as a result of strong heat release [56-58]. As the eigendirections 
(a)

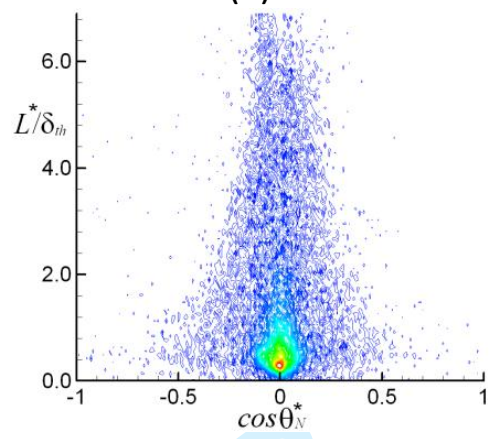

(c)

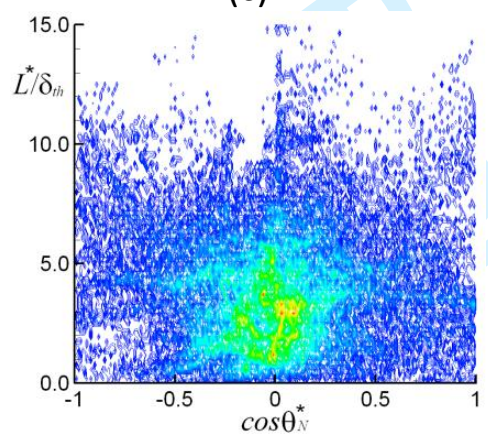

(b)

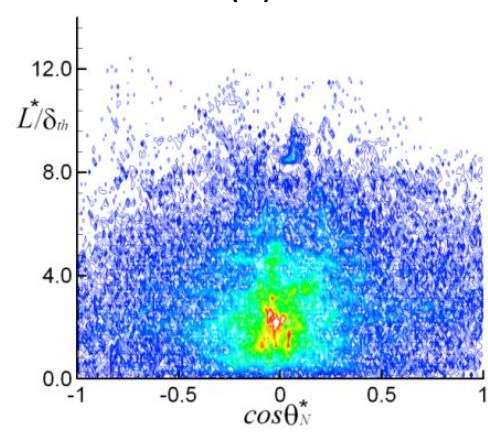

(d)

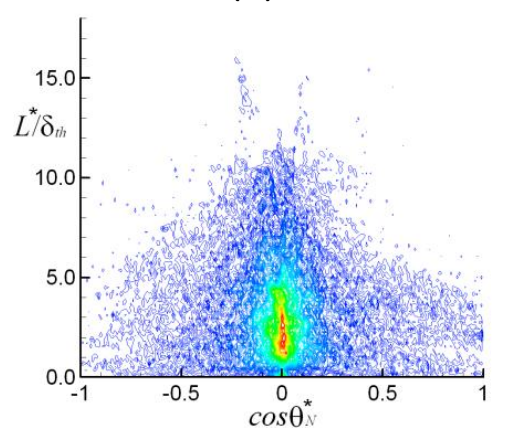

FIGURE 14. Joint PDFs between $L^{*}$ and $\cos \theta_{N}^{*}$ for $c=0.8$ in cases (a) A, (b) B, (c) C and (d) G.

The joint PDFs between $L^{*}$ and $\cos \theta_{N}^{*}$ for cases A, B, C and $\mathrm{G}$ are shown in Fig. 14. The overall tendency is the same as Fig. 13, but showing a much stronger clustering at $\cos \theta_{N}^{*} \approx 0$. It has already been shown in Fig. 7 that $\vec{\omega}^{*}$ strongly aligns with the intermediate principal strain rate for all cases, whereas $\vec{N}$ aligns with one of the other eigenvectors of the strain rate tensor. This gives rise to strong clustering at $\cos \theta_{N}^{*} \approx 0$ due to orthogonality of strain rate directions. Turbulent perturbations tend to produce short segments, whereas the heat release tends to produce large segments. The effects of flame generated turbulence are strong in case A, whereas turbulence decays across the flame in cases B-G $[29,43,59]$. The effects of flame-induced turbulence are strong in the negatively curved zones in case A due to strong flame normal acceleration arising from 
high dilatation rate. This high turbulence intensity in the negative curved zones in case A dominates over vorticity decay due to high dilatation rate to give rise to high probability of finding short vorticity segments in the negatively curved zones. By contrast, focusing of heat in the negatively curved zones leads to significant decay of vorticity and turbulent fluctuations in the negatively curved zones in cases B-G. This along with small values of dilatation rate in the positively curved zones in cases B-G gives rise to small vorticity segments in the positively curved regions.

(a)

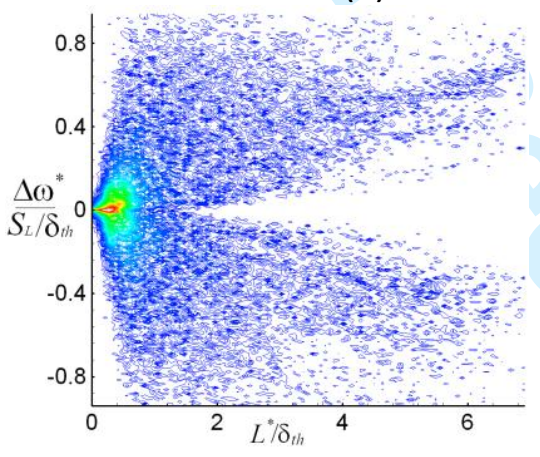

(c)

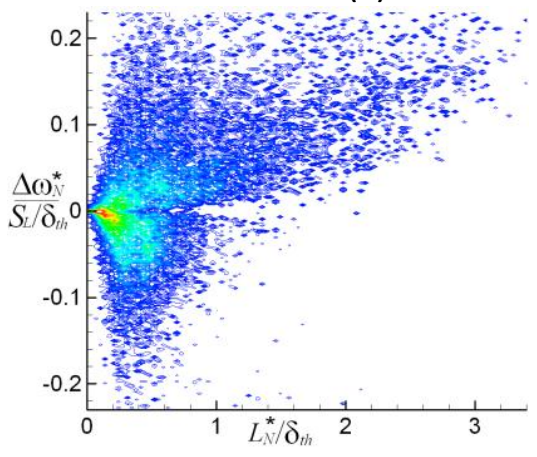

(b)

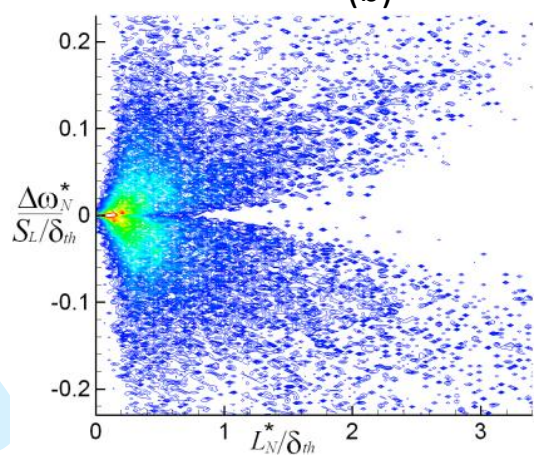

(d)

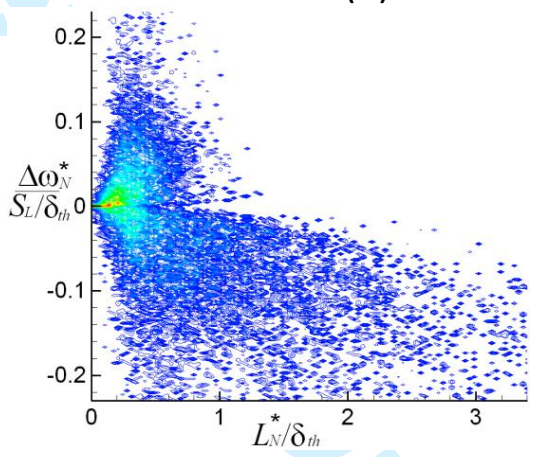

FIGURE 15. Joint PDF between (a) $\Delta \omega^{*}$ and $L^{*}$; (b) $\Delta \omega_{N}^{*}$ and $L_{N}^{*}$; (c) $\Delta \omega_{N}^{*}$ and $L_{N}^{*}$ conditional on VLS pointing to the unburnt side; (d) $\Delta \omega_{N}^{*}$ and $L_{N}^{*}$ conditional on VLS pointing to the burnt side for $c=0.8$ in case A.

As mentioned earlier, VLS can be characterized by the arc length and the vorticity difference between extremal points. In homogeneous non-reacting turbulence the joint PDF between $\Delta \omega$ and $L$ shows two distinct symmetrical branches for positive and negative VLS [14], which is in sharp contrast to the similar joint PDF for the 
velocity vector fields, where the branch corresponding to positive $\Delta u$ is larger in size than that of the negative $\Delta u$ part. Such a difference originates from different kinematic evolution tendencies of velocity and vorticity vectors. Interested readers are referred to Refs. [14,28] for the detailed discussion in this regard.

Similar to the non-reactive turbulence case, the joint PDFs between $\Delta \omega$ and $L$ in reactive turbulence are symmetric. For the $\vec{\omega}^{*}$ field, the joint PDF between $\Delta \omega^{*}$ and $L^{*}$ for case A is shown in Fig. 15 (a). Clearly even in reactive turbulence the symmetry between the branches with positive and negative $\Delta \omega^{*}$ is preserved. Such symmetry preservation in reactive turbulence can be understood as follows. Both positive VLS and negative VLS can point to either the burnt side or the unburnt side of the flame, which contributes to the positive and negative branches of the joint PDF without any particular preference to make it symmetric.

In order to understand the flame- turbulence interaction, it is important to account for the heat release effects, and the difference in (pseudo)vorticity statistics between the unburnt and burnt side of the flame. To serve this purpose, two new vector fields are defined as $\vec{\omega}_{N}^{*}=\left(\vec{\omega}^{*} \cdot \vec{N}\right) \vec{N}$ and $\vec{\omega}_{N}=(\vec{\omega} \cdot \vec{N}) \vec{N}$, which are the projection of $\vec{\omega}^{*}$ and $\vec{\omega}$ along the flame normal direction, respectively. Similarly VLS based on $\vec{\omega}_{N}^{*}$ $\left(\vec{\omega}_{N}\right)$ can be characterised using the arc length $L_{N}^{*}\left(L_{N}\right)$ and the scalar difference $\Delta \omega_{N}^{*}$ $\left(\Delta \omega_{N}\right)$. Figure 15(b) shows the joint PDF between $L_{N}^{*}$ and $\Delta \omega_{N}^{*}$ for case A, which is qualitatively the same as in Fig. 15(a). By grouping all VLS according to their orientations, VLS can point to either the unburnt side or the burnt side of the flame. The corresponding joint PDF conditional on VLS pointing to the unburnt side is shown in Fig. 15(c), while the joint PDF conditional on the VLS pointing to the burnt side is shown in Fig. 15(d). Very differently from Fig. 15 (a) and (b), the split joint PDFs in 
Fig. 15(c) and 15(d) are strongly asymmetric. The symmetry of Fig. 15(b) results from the superposition of Fig. 15 (c) and (d).

(a)

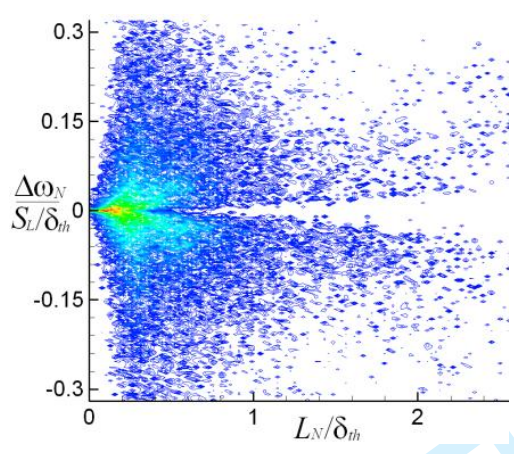

(b)

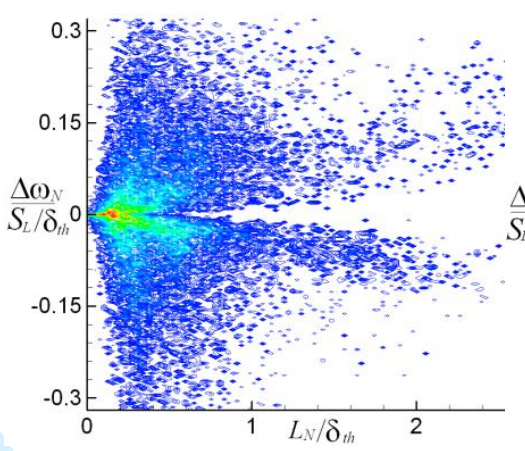

(c)

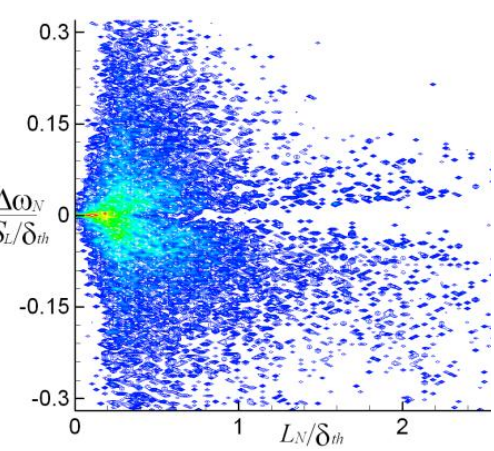

FIGURE 16. The joint PDF between $L_{N}$ and $\Delta \omega_{N}$ for $c=0.8$ in case A: (a) the overall result; (b) conditional on VLS pointing to the unburnt side; (c) conditional on VLS pointing to the burnt side.

For comparison the statistics of the VLS based on $\vec{\omega}_{N}$ are also calculated. Figure 16 (a) shows the overall joint PDF between $L_{N}$ and $\Delta \omega_{N}$, and Fig. 16 (b) and (c) are the joint PDFs of the same quantities but conditional on the VLS pointing to the unburnt side and burnt side, respectively. Interestingly, the split joint PDFs in Fig. 16 (b) and (c) are approximately symmetric, different from Fig. 15(c) and Fig. 15(d) for the $\vec{\omega}_{N}^{*}$ case. Results for case B are shown in Fig. 17 and Fig. 18. For the sake of brevity similar results for other cases are not presented.

From its definition, $\vec{\omega}_{N}^{*}\left(\vec{\omega}_{N}\right)$ is the component of pseudo-vorticity (vorticity) along the flame normal, which is determined by the velocity component tangential to the flame front. From the continuity relation, the tangential velocity component across the flame is unaltered, which explains the rough symmetry for the split joint PDFs in Figs. 16 and 18. The slight difference thereof can be due to the variation of the $\vec{\omega}_{N}$ strength across the flame. However, once weighted by density, the tangential velocity is 
(a)

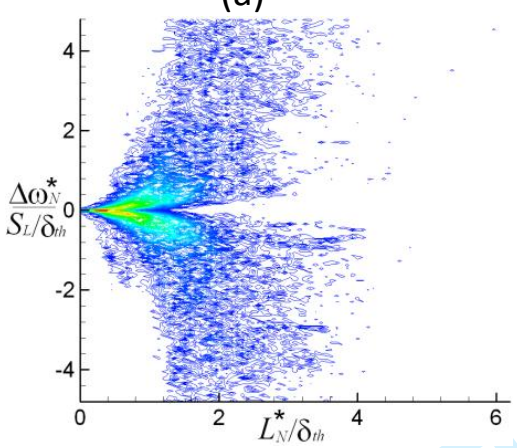

(b)

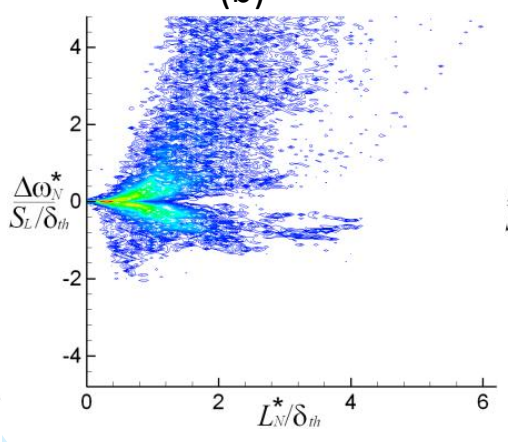

(c)

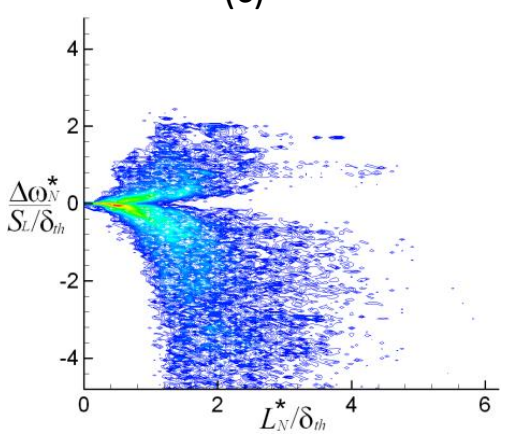

FiguRE 17. The joint PDF between $\Delta \omega_{N}^{*}$ and $L_{N}^{*}$ for $c=0.8$ in case B: (a) the overall result; (b) conditional on VLS pointing to the unburnt side; (c) conditional on VLS pointing to the burnt side.

(a)

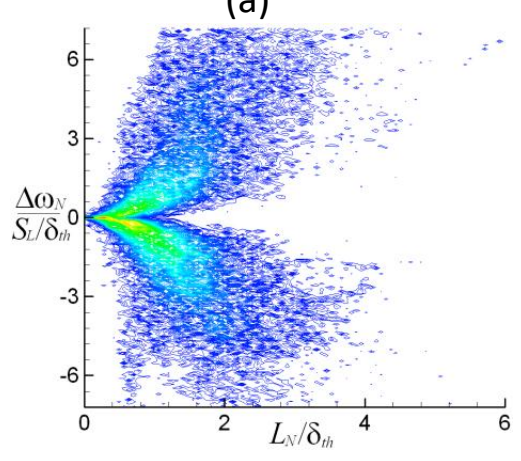

(b)

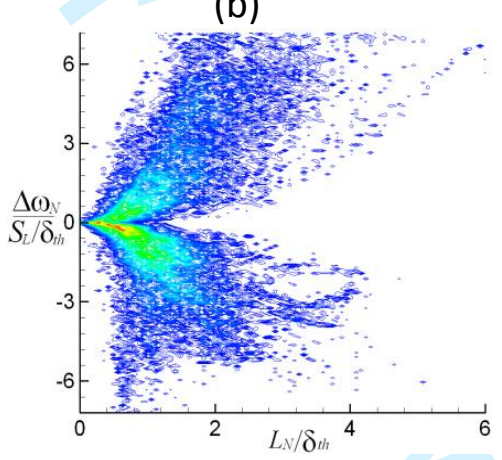

(c)

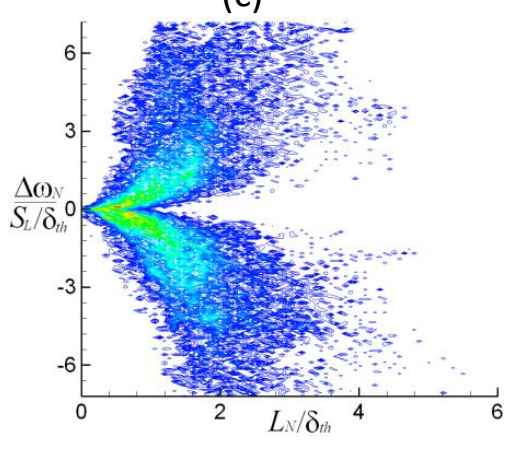

FIGURE 18. The joint PDF between $L_{N}$ and $\Delta \omega_{N}$ for $c=0.8$ in case B: (a) the overall result; (b) conditional on VLS pointing to the unburnt side; (c) conditional on VLS pointing to the burnt side.

A more detailed investigation of Figs. 15 and 17 reveals that the asymmetry has two root causes: (1) the variation of $\Delta \omega_{N}^{*}$ magnitude and (2) the variation of $L_{N}^{*}$ for positive and negative $\Delta \omega_{N}^{*}$ branches. The relative magnitudes of $u^{\prime} / S_{L}$ and heat release parameter $\tau$ determine the relative strength of thermal expansion in comparison to turbulent straining which is the strongest in case A and is becoming weaker from case A to case G. Thermal expansion tends to increase the VLS length and consequently Case A has the strongest asymmetry of $L_{N}^{*}$. The difference of $\Delta \omega_{N}^{*}$ for the positive and 
negative VLS is determined by the density jump across the flame. The value of $\tau$ increases from case A to case B to cases $\mathrm{C}-\mathrm{G}$, and therefore the asymmetry in $\Delta \omega_{N}^{*}$ increases in the same order.

In summary, the aforementioned joint PDFs provide statistical information about the primitive geometrical properties of the vorticity and pseudo-vorticity fields, for instance the eddy size $\left(\sim L\right.$ or $\left.L^{*}\right)$ and the vorticity fluctuation $\left(\sim \Delta \omega\right.$ or $\left.\Delta \omega^{*}\right)$. To address the different physical behaviour across the flame front, especially the components projected along the flame normal, i.e. $\vec{\omega}_{N}^{*}\left(\vec{\omega}_{N}\right)$, have been considered. Heat release imprints a strong asymmetry in the joint PDF between $L_{N}^{*}$ and $\Delta \omega_{N}^{*}$. Such non-local statistics depict how the flame interacts with near field turbulence. These effects should also be visible for the resolved scale quantities in the context of Large Eddy Simulations (LES) provided the sub-grid scale physics is properly accounted for.

\section{Conclusions}

The concept of pseudo-vorticity $\vec{\omega}^{*}$ (i.e. curl of density-weighted velocity vector $\rho \vec{u} / \rho_{0}$ ) has been introduced in this work and transport equations for $\vec{\omega}^{*}$ and pseudo-enstrophy (i.e. $\bar{\Omega}^{*}=\overline{\vec{\omega}^{*} \cdot \vec{\omega}^{*}} / 2$ ) have been derived. There is no difference between $\vec{\omega}$ and $\vec{\omega}^{*}$ for incompressible flows. However, the effects of density change within the flame due to heat release are explicitly accounted for in the definition of pseudo-vorticity $\vec{\omega}^{*}$. A detailed statistical analysis reveals that pseudo-vorticity exhibits more universal features compared to vorticity for a range of different conditions of turbulent premixed combustion. The statistical behaviour of vorticity $\vec{\omega}$ (i.e. curl of velocity vector $\vec{u}$ ) and pseudo-vorticity $\vec{\omega}^{*}$ (i.e. curl of density-weighted velocity vector $\rho \vec{u} / \rho_{0}$ ) have been investigated in detail based on a DNS database of freely propagating statistically planar flames with a range different values of $\mathrm{Da}$ and $\mathrm{Ka}$ spanning corrugated flamelets and 
thin reaction zones regimes of premixed turbulent combustion. It has been found that the relative orientation of $\vec{\omega}^{*}$ can be significantly different to that of $\vec{\omega}$ for small (large) values of $K a\left(R e_{t}\right)$. Furthermore, $\vec{\omega}^{*}$ has been found to align with the intermediate principal strain rate for all cases, which might be advantageous for modelling purposes, whereas $\vec{\omega}$ shows considerable alignment with either the most extensive or the most compressive principal strain rate depending on the case in addition to the predominant alignment with intermediate principal strain rate. The transport statistics of Reynolds averaged enstrophy (i.e. $\bar{\Omega}=\overline{\vec{\omega} \cdot \vec{\omega}} / 2$ ) and pseudo-enstrophy (i.e. $\bar{\Omega}^{*}=\overline{\vec{\omega}}^{*} \cdot \vec{\omega}^{*} / 2$ ) have been investigated in detail. The transport characteristics of $\bar{\Omega}^{*}$ remain mostly unchanged for the cases analysed here. Thus, the apparently universal feature of pseudo-vorticity can be advantageous for the purpose of developing closure strategies for the dissipation of turbulent kinetic energy (i.e. $\left.\tilde{\varepsilon}=\overline{\mu\left(\partial u_{l}^{\prime \prime} / \partial x_{\jmath}\right)\left(\partial u_{l}^{\prime \prime} / \partial x_{\jmath}\right)} / \bar{\rho}\right)$ in terms of enstrophy based on pseudo-vorticity (i.e. $\bar{\rho} \tilde{\varepsilon} \sim \mu \bar{\Omega}^{*}$ ).

The non-local vorticity statistics in turbulent premixed combustion have been investigated in terms of the VLS structure, which can be characterised by the segment arc length and the difference in vorticity magnitude across the segment. The joint PDF between the two characteristic parameters inherits important imprint of flameturbulence interaction. By defining the VLS based on the $\vec{\omega}_{N}^{*}$ field, the conditional statistics for VLS pointing to the unburnt and burnt side are largely different. Physically if the effects of dilatation rate are relatively strong, e.g. in case A, the difference is mainly from the length of VLS, whereas if effects of dilatation are relatively weak, i.e. the flow is closer to a non-reactive case, the observed asymmetry in the joint PDFs is mainly from the difference of $\Delta \omega_{N}^{*}$, which can be attributed to the density jump across the flame. As the alignment of vorticity with local principal strain rates, and the statistical behaviour of VLS change from one case to another, whereas the 
corresponding statistics for pseudo-vorticity does not alter for the flames with widely different values of Damköhler and Karlovitz numbers, the concept of pseudo-vorticity offers potential advantages for the analysis of compressible reacting flows over conventional vorticity. Exemplarily, pseudo-vorticity in reacting flows behaves in some aspects similar to vorticity in isothermal flows and consequently it might be possible to use well-established modelling concepts. Furthermore, the absence of pressure in the pseudo-vorticity has the potential to offer advantages in the compressible flow problems with pressure discontinuities (e.g. shock, detonation etc.). A preliminary analysis by these authors using a detailed chemistry DNS database by Im et al. [60], involving two cases representing typical flow situations in the corrugated flamelets and thin reaction zones regimes, revealed that the qualitative nature of the statistical behaviour of vorticity $\vec{\omega}$ and pseudo-vorticity $\vec{\omega}^{*}$ do not change due to detailed chemistry and transport but further investigation for higher values of $R e_{t}$ will be necessary for more comprehensive physical understanding.

Acknowledgements: NC is grateful to the Engineering and Physical Sciences Research Council (EPSRC) and N8/ARCHER for financial and computational supports respectively. LW acknowledges the funding support by National Science Foundation China (NSFC) under the grant number 91441116. NC and MK are grateful to Prof. H.G. Im from KAUST, Saudi Arabia for giving access to his DNS database.

\section{Appendix A}

In order to derive a transport equation for pseudo-vorticity $\omega_{i}^{*}=\rho \omega_{i} / \rho_{0}+$ $\left(\epsilon_{i j k} / \rho_{0}\right)\left(\partial \rho / \partial x_{j}\right) u_{k}$ a transport equation for each individual component will be required. Using the product rule of differentiation the continuity equation reads

$$
\frac{\partial \rho}{\partial t}+u_{l} \frac{\partial \rho}{\partial x_{l}}+\rho \frac{\partial u_{l}}{\partial x_{l}}=0
$$


Multiplying the vorticity transport Equation (2) with density and Eq. (A1) with vorticity $\omega_{i}$ and adding them together gives the following expression for $\frac{D}{D t} \rho \omega_{i}$ :

$$
\begin{gathered}
\frac{\partial}{\partial t}\left(\rho \omega_{i}\right)+u_{l} \frac{\partial}{\partial x_{l}}\left(\rho \omega_{i}\right)=\rho \omega_{l} \frac{\partial u_{i}}{\partial x_{l}}-\frac{\epsilon_{i j k}}{\rho} \frac{\partial \rho}{\partial x_{j}} \frac{\partial \tau_{k l}}{\partial x_{l}}+\epsilon_{i j k} \frac{\partial^{2} \tau_{k l}}{\partial x_{j} \partial x_{l}} \\
-2 \rho \omega_{i} \frac{\partial u_{l}}{\partial x_{l}}+\frac{\epsilon_{i j k}}{\rho} \frac{\partial \rho}{\partial x_{j}} \frac{\partial p}{\partial x_{k}}
\end{gathered}
$$

Taking next the partial derivative $\partial / \partial x_{j}$ of Eq. (A1) and multiplying the result with $u_{k}$ yields

$$
u_{k} \frac{\partial}{\partial t}\left(\frac{\partial \rho}{\partial x_{j}}\right)+u_{k} u_{l} \frac{\partial}{\partial x_{j}}\left(\frac{\partial \rho}{\partial x_{l}}\right)=-u_{k} \frac{\partial u_{l}}{\partial x_{j}} \frac{\partial \rho}{\partial x_{l}}-u_{k} \frac{\partial \rho}{\partial x_{j}} \frac{\partial u_{l}}{\partial x_{l}}-\rho u_{k} \frac{\partial^{2} u_{l}}{\partial x_{j} \partial x_{l}}
$$

Multiplying the momentum equation (1) with $\partial \rho / \partial x_{j}$ results in

$$
\frac{\partial \rho}{\partial x_{j}} \frac{\partial u_{k}}{\partial t}+u_{l} \frac{\partial \rho}{\partial x_{j}} \frac{\partial u_{k}}{\partial x_{l}}=-\frac{1}{\rho} \frac{\partial \rho}{\partial x_{j}} \frac{\partial p}{\partial x_{k}}+\frac{1}{\rho} \frac{\partial \rho}{\partial x_{j}} \frac{\partial \tau_{k l}}{\partial x_{l}}
$$

Adding Eqs. (A3) and (A4) and subsequently taking curl an expression for transport of $\epsilon_{i j k}\left(\partial \rho / \partial x_{j}\right) u_{k}$ is obtained:

$$
\begin{gathered}
\frac{\partial}{\partial t}\left(\epsilon_{i j k} \frac{\partial \rho}{\partial x_{j}} u_{k}\right)+u_{l} \frac{\partial}{\partial x_{l}}\left(\epsilon_{i j k} \frac{\partial \rho}{\partial x_{j}} u_{k}\right)=-\epsilon_{i j k} u_{k} \frac{\partial u_{l}}{\partial x_{j}} \frac{\partial \rho}{\partial x_{l}}-\epsilon_{i j k} u_{k} \frac{\partial \rho}{\partial x_{j}} \frac{\partial u_{l}}{\partial x_{l}} \\
-\epsilon_{i j k} \rho u_{k} \frac{\partial^{2} u_{l}}{\partial x_{j} \partial x_{l}}-\frac{\epsilon_{i j k}}{\rho} \frac{\partial \rho}{\partial x_{j}} \frac{\partial p}{\partial x_{k}}+\frac{\epsilon_{i j k}}{\rho} \frac{\partial \rho}{\partial x_{j}} \frac{\partial \tau_{k l}}{\partial x_{l}}
\end{gathered}
$$

After multiplication with $1 / \rho_{0}$ Eq. (A2) and Eq. (A5) can be combined to yield Eq. 5:

$$
\begin{aligned}
& \frac{\partial \omega_{i}^{*}}{\partial t}+u_{k} \frac{\partial \omega_{i}^{*}}{\partial x_{k}}=\frac{\rho}{\rho_{0}} \omega_{k} \frac{\partial u_{i}}{\partial x_{k}}-\omega_{i}^{*} \frac{\partial u_{k}}{\partial x_{k}}-\frac{\rho}{\rho_{0}} \omega_{i} \frac{\partial u_{k}}{\partial x_{k}}+\frac{\epsilon_{i j k}}{\rho_{0}} \frac{\partial^{2} \tau_{k l}}{\partial x_{j} \partial x_{l}}-\frac{\epsilon_{i j k}}{\rho_{0}} u_{k} \frac{\partial u_{l}}{\partial x_{j}} \frac{\partial \rho}{\partial x_{l}}- \\
& \epsilon_{i j k} \frac{\rho}{\rho_{0}} u_{k} \frac{\partial^{2} u_{l}}{\partial x_{j} \partial x_{l}}
\end{aligned}
$$

\section{REFERENCES}

[1] Davidson PA. Turbulence: An introduction for scientists and engineers, Oxford University Press; 2004. 
[2] She Z, Jackson E, Orszag SA. Intermittent vortex structures in homogeneous isotropic turbulence, Nature 1990; 344, 226.

[3] Tsinober A. An informal introduction to turbulence, Kluwer; 2001.

[4] Moffatt HK. \& Tsinober A. Helicity in laminar and turbulent flow, Annu. Rev. Fluid Mech. 1992; 24, 281.

[5] Synge JL, Lin CC. On a statistical model of isotropic turbulence, Trans.R.Soc. Can. $1943 ; 37,45$.

[6] Aivasis K, Pullin D. On velocity structure functions and the spherical vortex model for isotropic turbulence, Phys. Fluids 2001; 13(7): 2019.

[7] Ruetsch GR, Maxey MR. Small-scale features of vorticity and passive scalar fields inhomogeneous isotropic turbulence, Phys. Fluids A 1991; 3(6), 1587.

[8] Townsend A. On the fine structure of turbulence, Proc.R.Soc.Lond, A 1951; 208, 534.

[9] Horiuti K, Fujisawa T. The multi-mode stretched spiral vortex in homogeneous isotropic turbulence, J. Fluid Mech. 2008; 595, 341.

[10] Jimenez J, WRAY AA. On the characteristics of vortex filaments in isotropic turbulence, J. Fluid Mech. 1998; 373, 255.

[11] Tennekes H, Lumley JL. A first course in turbulence, 1st Edition, MIT Press, Cambridge, Massachusetts, USA; 1972.

[12] Haller G. An objective definition of a vortex, J. Fluid Mech. 2005; 525, 1.

[13] Kolar V. Vortex identification: New requirements and limitations, Int. J. Heat and FluidFlow 2007; 28, 638.

[14] Wang, L. Structures of the vorticity tube segment in turbulence. Physics of Fluids $2012 ; 24,045101$. 
[15] Nomura KK, Elghobashi SE. The structure of inhomogeneous turbulence in variable density nonpremixed flames, Theor. Fluid Dyn. 1993; 5, 153.

[16] Boratov N. Elghobashi SE, Zhong R. On the alignment of strain, vorticity and scalar gradient in turbulent, buoyant, nonpremixed flames, Phys. Fluids 1996; 10(9), 2260 .

[17] Jaberi FA, Livescu D,. Madnia CK. Characteristics of chemically reacting compressible homogeneous turbulence, Phys. Fluids 2000; 12 (5), 1189.

[18] Hamlington PE, Poludnenko AY, Oran ES. Interactions between turbulence and flames in premixed reacting flows, Phys. Fluids 2011; 23, 125111.

[19] Chomiak J, Nisbet J. Modelling variable density effects in turbulent flames, Combust. Flame 1995; 102, 371.

[20] Chakraborty N. Statistics of vorticity alignment with local strain rates in turbulent premixed flames, Eur. J. Mech. B/Fluids 2014; 46, 201.

[21] Treurniet TC, Nieuwstadt FTM, Boersma BJ. Direct numerical simulation of homogeneous turbulence in combination with premixed combustion at low Mach number modelled by the G-equation, J. Fluid Mech. 2006; 565, 25.

[22] Lipatnikov AN, Nishiki S, Hasegawa T. A direct numerical study of vorticity transformation in weakly turbulent premixed flames, Phys. Fluids 2014; 26, 105104.

[23] Steinberg AM, Driscoll JF, Ceccio SL. Measurements of turbulent premixed flame dynamics using cinema stereoscopic PIV, Exp. Fluids 2008; 44, 985.

[24] Steinberg AM, Driscoll JF. Straining and wrinkling processes during turbulencepremixed flame interaction measured using temporally-resolved diagnostics, Combust. Flame 2009; 156, 2285. 
[25] Steinberg AM, Driscoll JF, Ceccio SL. Temporal evolution of flame stretch due to turbulence and the hydrodynamic instability, Proc. Combust. Inst. 2009; 32, 1713.

[26] Steinberg AM, Driscoll JF, Ceccio SL. Three-dimensional temporally resolved measurements of turbulence-flame interactions using orthogonal-plane cinemastereoscopic PIV, Exp. Fluids 2009; 47, 527.

[27] Wang L, Peters N. Length scale distribution functions and conditional means for various fields in turbulence, J. Fluid Mech. 2006; 554, 457.

[28] Wang L. On properties of fluid turbulence along streamlines, J. Fluid Mech. 2010; $648,183$.

[29] Wang L., Chakraborty N, Zhang J. Streamline segment analysis of turbulent premixed flames, Proc. Combust. Inst. 2013; 34, 1401.

[30] Chakraborty N, Wang L, Klein M. Streamline segment statistics of premixed flames with non-unity lewis numbers, Physical Rev. E 2014; 89, 033015.

[31] Chen JH, Choudhary A, de Supinski B. et al. Terascale direct numerical simulations of turbulent combustion using S3D, Comp. Sci. Discov. 2009; 2, 015001.

[32] Rutland CJ, Cant RS. Turbulent transport in premixed flames, Proc.1994 Summer Program, Center for Turbulence Research 1994; 75-94.

[33] Jenkins KW, Cant RS. DNS of turbulent flame kernels, in Proc. 2nd AFOSR Conference on DNS and LES, (Kluwer Academic Publishers (Dordrecht); 1999, p. 192.

[34] Poinsot T, Lele SK. Boundary conditions for direct simulation of compressible viscous flows, J. Comp. Phys. 1992; 101, 104.

[35] Rogallo RS. Numerical experiments in homogeneous turbulence, NASA Technical Memorandum 91416, NASA Ames Research Center, California, 1981. 
[36] Veynante D, Trouvé A., Bray KNC. et al. Gradient and counter-gradient turbulent scalar transport in turbulent premixed flames, J. Fluid Mech. 1997; 332, 263.

[37] Boger M., Veynante D., Boughanem H, et. Al. A. Direct Numerical Simulation analysis of flame surface density concept for Large Eddy Simulation of turbulent premixed combustion, Proc. Combust. Inst. 1998; 27, 917.

[38] Charlette F, Meneveau C, Veynante D. A power-law flame wrinkling model for LES of premixed turbulent combustion. Part I: Nondynamic formulation and initial tests, Combust. Flame 2002; 131, 181.

[39] Swaminathan N, Bray KNC. Effect of dilatation on scalar dissipation in turbulent premixed flames, Combust. Flame 2005; 143, 549.

[40] Swaminathan N, Grout R. Interaction of turbulence and scalar fields in premixed flames, Phys. Fluids 2006; 18, 045102.

[41] Grout R. An age extended progress variable for conditioned reaction rates, Phys. Fluids 2007; 19, 105107.

[42] Han I, Huh KH. Effects of Karlovitz number on the evolution of the flame surface density in turbulent premixed flames, Proc. Combust. Inst. 2009; 32, 1419.

[43] Chakraborty N, Katragadda M, Cant RS. Statistics and modelling of turbulent kinetic energy transport in different regimes of premixed combustion, Flow Turb. Combust. 2011; 87,205.

[44] Chakraborty N, Hartung G, Katragadda M. et al. A numerical comparison of 2D and 3D density-weighted displacement speed statistics and implications for laser based measurements of flame displacement speed, Combust. Flame 2011; 158, 1372.

[45] Bray KNC. Libby PA, Moss JB. Unified modelling approach for premixed turbulent combustion - Part I: General formulation, Combust. Flame 1985; 61, 87. 
[46] Chakraborty N. Comparison of displacement speed statistics of turbulent premixed flames in the regimes representing combustion in corrugated flamelets and thin reaction zones, Phys. Fluids 2007; 19, 105109.

[47] Bychkov V. Nonlinear equation for a curved stationary flame and flame velocity, Phys. of Fluids, A 1998; 10,8, 2091.

[48] Chakraborty N, Cant RS. Effects of Strain Rate and Curvature on Surface Density Function transport in Turbulent Premixed Flames in the Thin Reaction Zones Regime, Phys. Fluids 2005; 17, 105105.

[49] Chakraborty N, Hawkes ER, Chen JH, et al. Effects of strain rate and curvature on Surface Density Function transport in turbulent premixed CH4-air and H2-air flames: A comparative study, Combust. Flame 2008; 154, 259.

[50] Chakraborty N, Klein M. Influence of Lewis number on the Surface Density Function transport in the thin reaction zones regime for turbulent premixed flames, Phys. Fluids 2008; 20, 065102, 1.

[51] Chong MS, Perry AE, Cantwell BJ. A general classification of three dimensional flow patterns. Physics of Fluids A 1990; 2 (5), 765.

[52] Cifuentes L, Dopazo C, Martin J et al. Local flow topologies and scalar structures in a turbulent premixed flame, Phys. Fluids 2014; 26, 065108.

[53] Ashurst W, Kerstein A, Kerr RM. \& Gibson CH. Alignment of vorticity and scalar gradient with strain rate in simulated Navier-Stokes turbulence, Phys. Fluids A 1987; 30, 2343.

[54] Majda AJ. Vorticity, turbulence, and acoustics in fluid flow, SIAM Rev. 1991; 33, 349.

[55] Jimenez J. Kinematic alignment effects in turbulent flows, Phys. Fluids A 1992; 4, 652. 
[56] Chakraborty N, Swaminathan N. Influence of Damköhler number on turbulencescalar interaction in premixed flames, Part I: Physical Insight, Phys. Fluids 2007; 19, 045103.

[57] Hartung G, Hult J, Kaminski C.F. et al. Effect of heat release on turbulence and scalar-turbulence interaction in premixed combustion, Phys. Fluids 2008; 20. 035110.

[58] Steinberg AM, Driscoll JF, Swaminathan N. Statistics and dynamics of turbulenceflame alignment in premixed combustion, Combust. Flame 2012; 159, 2576.

[59] Chakraborty N, Cant RS. Effects of turbulent Reynolds number on the modelling of turbulent scalar flux in premixed flames. Numerical Heat Transfer A 2015; 67(11), 1187-1207.

[60] Im, H.G., Arias, P.G., Chaudhuri, S., Uranakara, H.A. 2016 Direct numerical simulations of statistically stationary turbulent premixed flames, Combust. Sci. Technol., in press. 